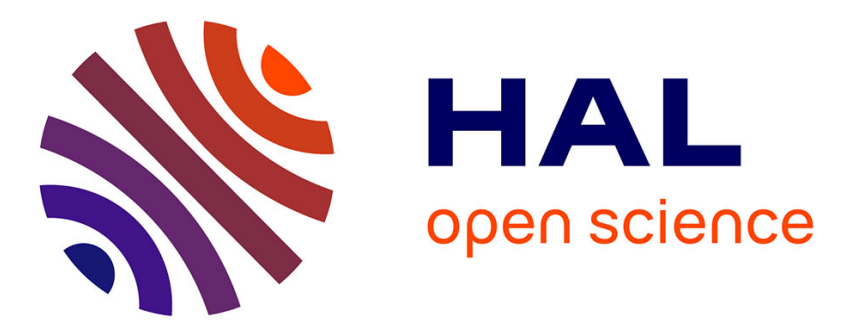

\title{
NUMERICAL MODELS OF SATURN'S LONG-LIVED ANTICYCLONES
}

\author{
Enrique García-Melendo, Agustín Sánchez-Lavega, Ricardo Hueso
}

\section{To cite this version:}

Enrique García-Melendo, Agustín Sánchez-Lavega, Ricardo Hueso. NUMERICAL MODELS OF SATURN'S LONG-LIVED ANTICYCLONES. Icarus, 2007, 191 (2), pp.665. 10.1016/j.icarus.2007.05.020 . hal-00499076

\section{HAL Id: hal-00499076 https://hal.science/hal-00499076}

Submitted on 9 Jul 2010

HAL is a multi-disciplinary open access archive for the deposit and dissemination of scientific research documents, whether they are published or not. The documents may come from teaching and research institutions in France or abroad, or from public or private research centers.
L'archive ouverte pluridisciplinaire HAL, est destinée au dépôt et à la diffusion de documents scientifiques de niveau recherche, publiés ou non, émanant des établissements d'enseignement et de recherche français ou étrangers, des laboratoires publics ou privés. 


\section{Accepted Manuscript}

\section{NUMERICAL MODELS OF SATURN'S LONG-LIVED ANTICYCLONES}

Enrique García-Melendo, Agustín Sánchez-Lavega, Ricardo Hueso

PII: $\quad$ S0019-1035(07)00271-0

DOI: $\quad$ 10.1016/j.icarus.2007.05.020

Reference: $\quad$ YICAR 8318

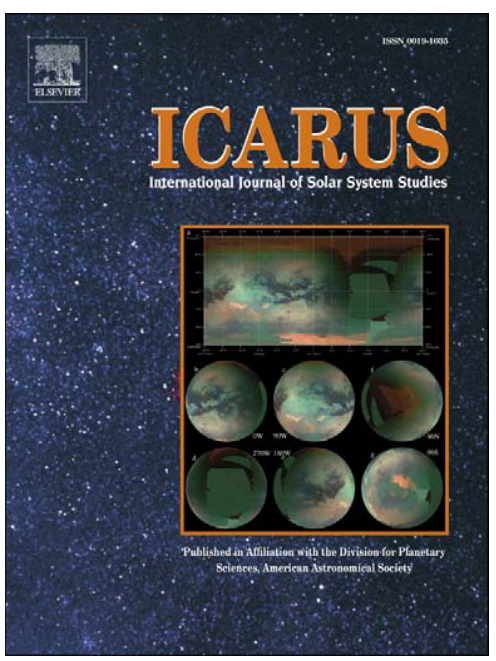

To appear in: Icarus

Received date: 12 April 2006

Revised date: 3 May 2007

Accepted date: 28 May 2007

Please cite this article as: E. García-Melendo, A. Sánchez-Lavega, R. Hueso, NUMERICAL MODELS OF SATURN'S LONG-LIVED ANTICYCLONES, Icarus (2007), doi: 10.1016/j.icarus.2007.05.020

This is a PDF file of an unedited manuscript that has been accepted for publication. As a service to our customers we are providing this early version of the manuscript. The manuscript will undergo copyediting, typesetting, and review of the resulting proof before it is published in its final form. Please note that during the production process errors may be discovered which could affect the content, and all legal disclaimers that apply to the journal pertain. 


\title{
NUMERICAL MODELS OF SATURN'S LONG-LIVED ANTICYCLONES
}

\author{
Enrique García-Melendo \\ Esteve Duran Observatory Foundation \\ Seva, Spain \\ Agustín Sánchez-Lavega
}

Dpto. Física Aplicada I, Escuela Superior de Ingeniería, Universidad del País Vasco, Bilbao, Spain

\section{Ricardo Hueso}

Dpto. Física Aplicada I, Escuela Superior de Ingeniería, Universidad del País Vasco, Bilbao, Spain

\section{Submitted to Icarus}

Received

Revised

Number of pages: 48

Number of Figures: 11

Number of Tables: 3 
Proposed Running Head: Saturn's Anticyclones

\section{Editorial correspondence to:}

Dr. Enrique García-Melendo

Esteve Duran Observatory Foundation

Montseny 42

085330 Seva

Spain

E-mail: duranobs@astrogea.org 


\section{Abstract}

New measurements of the dynamical properties of the long-lived Saturn's anticyclonic vortex known as "Brown Spot" (BS), discovered during the Voyager 1 and 2 flybys in 1980-81 at latitude $43.1^{\circ} \mathrm{N}$, and model simulations using the EPIC code, have allowed us to constrain the vertical wind shear and static stability in Saturn's atmosphere (vertically from pressure levels from 10 mbar to 10 bars) at this latitude. BS dynamical parameters from Voyager images include its size as derived from cloud albedo gradient (6100 km East-West times $4300 \mathrm{~km}$ North-South), mean tangential velocity (45 \pm 11 $\mathrm{ms}^{-1}$ at $2400 \mathrm{~km}$ from centre) and mean vorticity $\left(4.0 \pm 1.5 \times 10^{-5} \mathrm{~s}^{-1}\right)$, lifetime $>1$ year, drift velocity $\left(5.3 \pm 0.1 \mathrm{~ms}^{-1}\right)$ relative to Voyager's System III rotation rate, mean meridional atmospheric wind profile at cloud level at its latitude and interactions with nearby vortices (pair orbiting and merging). An extensive set of numerical experiments have been performed to try to reproduce this single vortex properties and its observed mergers with smaller anticyclones by varying the vertical structure of the zonal wind and adjusting the static stability of the lower stratosphere and upper troposphere. Within the context of the EPIC model atmosphere, our simulations indicate that BS's drift velocity, longevity and merging behaviour are very sensitive to these two atmospheric properties. The best results at the BS latitude occur for static stability conditions that use a Brunt-Väisäla frequency constant in the upper troposphere (from 0.5 to 10 bar) above $3.2 \times 10^{-3} \mathrm{~s}^{-1}$ and suggest that the wind speed slightly decays below the visible cloud deck from $\sim 0.5$ to 10 bar at a rate $\partial \mathrm{u} / \partial \mathrm{z} \sim 2-6 \mathrm{~ms}^{-1}$ per scale height. Changing the vortex latitude within the band domain introduces latitude oscillations in the vortex but not a significant meridional migration. Simulated mergers always showed orbiting 
movements with a typical merging time of about three days, very close to the time-span observed in the interaction of real vortices. Although these results are not unique in view of the unknowns of Saturn's deep atmosphere, they serve to constrain realistically its structure for ongoing Cassini observations.

\section{Introduction}

The Voyagers 1 and 2 flybys of Saturn in 1980 and 1981 revealed the existence of a variety of "spots" with different sizes and contrasts distributed in a wide range of latitudes (Smith et al., 1981, 1982; Ingersoll et al., 1984). Among them, there was a conspicuous group of oval vortices that resembled in their aspect and longevity those found in Jupiter (Sromovsky et al., 1983; Ingersoll et al., 1984; Sánchez-Lavega et al., 1997, 2000). Most of them were anticyclones, as derived from their internal rotation speed, with the largest reaching East-West dimensions between 5000 and $10000 \mathrm{~km}$. Particularly relevant were the so called "brown spots" (abbreviated in what follows as BS) located at latitude $\sim 43^{\circ}$ North within a domain of anticyclonic wind shear (Sromovsky et al., 1983; Ingersoll et al., 1984; Sánchez-Lavega et al., 2000). The most conspicuous among them had about 5000 by $3000 \mathrm{~km}$, with anticyclonic circumferential flow of $30 \mathrm{~ms}^{-1}$ and a mean vorticity of $5 \times 10^{-5} \mathrm{~s}^{-1}$ (Smith et al., 1982). Sromovsky et al. (1983) tracked these features on Voyager 1 images during $\sim 20$ days measuring their

zonal velocity with averaged value $4.4 \pm 1 \mathrm{~ms}^{-1}$ relative to System III rotation frame (Desh and Kaiser, 1981). A first reanalysis of the motion of this vortex from Voyager 1 and 2 was performed by Sánchez-Lavega et al. (2000) extending the tracking to 1 year (including Voyager 1 and 2 data). In this paper we extend this analysis to the highest 
resolution Voyager 2 images. On the other hand, the latitude band where BS formed was particularly active dynamically because of the existence of numerous other smaller spots (oval and irregular in shape) that interacted, colliding and merging after mutual orbiting (Smith et al., 1982; Sromovsky et al., 1983).

No counterpart of this kind of spots and activity was reported at this epoch in the southern hemisphere, whose visibility from Voyager spacecrafts was worse. However, this hemisphere was inspected with the Hubble Space Telescope since early 1990s at lower resolution, but still enough to detect the larger and contrasted spots. From 1994 to 2004 we recorded in the southern anticyclonic domain, between latitudes $40^{\circ} \mathrm{S}$ to $44^{\circ} \mathrm{S}$ and symmetrically with respect to the northern latitudes, series of dark spots that remember the "brown spots", the largest with $\sim 4000 \mathrm{~km}$ and moving with speeds between 1 and $-5 \mathrm{~ms}^{-1}$ (Sánchez-Lavega et al. 2003, 2004). These dark spots were later captured by Cassini ISS images before the spacecraft orbit insertion in July 2004, showing mutual merging behaviour when two similar spots approached (Porco et al., 2005). Vasavada et al. (2006) observed them at high-resolution using Cassini images from March-September 2004. The visual aspect and size of the two vortices present in September 2004 were similar to those of BS being probably anticyclones according to the zonal wind profile at their latitude. In addition this latitude band is prolific in forming white storms, probably convective events, observed with HST (SánchezLavega et al., 2003, 2004) and Cassini ISS (Porco et al., 2005; Vasavada et al., 2006). In view of the knowledge of their main properties and similarities, brown spots in the northern and southern hemispheres are at present the best candidates for the study of the dynamics of anticyclones in Saturn, and their properties can be used to constraint the dynamical structure of the upper troposphere. 
A variety of models have been previously used to study the longevity, stability, motions and mergers of Jovian anticyclones using a two-dimensional quasigeostrophic models and three-dimensional baroclinic multilayer models (see Ingersoll et al., 1984, 2004; and for a recent review Vasavada and Showman, 2005). We apply a similar threedimensional baroclinic multilayer model to simulate a Saturnian BS.

This paper deals with the report of new and improved measurements of a brown spot's main properties seen at the Voyager epoch, and used as input to perform numerical simulation of its basic characteristics and of their mutual interactions (mergers). Our aim is to use BS properties to model Saturn's anticyclones and to use BS as a probe of the dynamical state of Saturn's lower stratosphere and upper troposphere in its latitude (from 10 mbar down to about 10 bar where water clouds are theoretically predicted). We use for this purpose the EPIC code, an Atmospheric General Circulation Model well tested in previous studies of the giant planets (Dowling et al., 1998). A similar exercise was previously performed in Jupiter (Morales-Juberias et al., 2003).

\section{Properties of the Brown Spot}

Our study used archived images of Saturn obtained by the spacecraft Voyager 2 from 15 to 24 August 1981. The Voyager ISS instrument (Imaging Science System) onboard the Voyager spacecraft used wide angle (WA) and narrow angle (NA) Vidicon cameras (see Smith et al. 1977, for details on the cameras and filters). Copies of most of the images were acquired on CD-ROMs from the Planetary Data System (PDS) deep

archive located at the National Space Science Data Center (NSSDC). Table 1 gives the list and dates of the images we have used. 


\section{[Table 1]}

The image selection was based on searching for the highest-resolution images of the vortex BS in image pairs showing the same area with a minimum temporal separation $\sim 10 \mathrm{hr}$. Measurements were performed on green filtered images (effective wavelength of $566 \mathrm{~nm}$ ) because they showed the clouds with higher contrast than in other filters, at a typical spatial resolution $\sim 25-30 \mathrm{~km} /$ vidicon line pair. The procedure to measure positions on Voyager images is fully described in Sánchez-Lavega et al. (2000) and Legarreta and Sanchez-Lavega (2005), so we do not repeat it here. In Figure 1 we present the visual aspect of BS1 identifying the main features used as tracers of the BS flow. Wind velocity measurements were obtained by tracking the position of individual cloud features in the vortex area. Wind velocities were measured relative to Voyagers' System III radio rotation period (Desh and Kaiser, 1981), but note that the determination of the true rotation period of Saturn is still under debate (within a range of \pm 7-8 minutes) in view of Ulysses and Cassini observations (Gurnett et al., 2007 and references therein). The size and number of the cloud elements used as tracers on the vortex were scarce and only 13 features were identified moving around BS. However, many others were tracked at other longitudes at the BS latitude allowing to retrieve the zonal wind profile. The problem resides in that Saturn's anticyclones show filamentary stream-like features in their interior with few single isolated spots (as compared e. g. to Jupiter), a behaviour confirmed by Cassini images (Vasavada et al., 2006). The track of these features was manually performed on the side-by-side (or blinking) pair of images (Figure 1).

[Figure 1] 
The green filter (central wavelength $566 \mathrm{~nm}$ ) shows BS cloud morphology divided in two parts: an oval dark central core $(950 \mathrm{~km}$ in zonal length and $700 \mathrm{~km}$ meridional width) surrounded by a lighter oval area (enclosed by bright filaments) that define the vortex outer edge. This are has a nearly elliptical shape with semi-major axis a $=3025 \pm$ $100 \mathrm{~km}$ and semi-minor axis $\mathrm{b}=2120 \pm 100 \mathrm{~km}$ corresponding to a flattening $\varepsilon=1-\mathrm{b} / \mathrm{a}$ $=0.29\left(\right.$ or alternatively to an eccentricity e $\left.=\left(1-\mathrm{b}^{2} / \mathrm{a}^{2}\right)^{1 / 2}=0.71\right)$. The vortex eccentricity changed with time (compare the two images in Figure 1) although preserving always the elliptical shape. The vortex size as seen by cloud albedo contrast is coincident within a $5 \%$ uncertainty with the motions traced by the streamlines in the fluid. In order to measure the flow around BS we first identified the vortex centre by tracing the ellipse semi-major and -minor axis. We found BS centre to be at latitude $43.1^{\circ} \pm 0.4^{\circ} \mathrm{N}$. The second step was to measure in each image the polar position $(r, \theta)$ of the flow features relative to BS centre.

To measure the tangential velocity of each tracer, we calculate the length of the arc of the ellipse described by them during the tracking, assuming they moved following an elliptical path with the given eccentricity and semi-axes $(a, b)$. The length of this arc is given by

$$
\Delta s=\int_{\theta_{1}}^{\theta_{2}} E(\theta, \varepsilon) d \theta
$$

where $\Delta \mathrm{s}$ is an incomplete elliptic integral of the second kind and

$$
E(\theta, \varepsilon)=\left[\left(\frac{r\left(2 \varepsilon-\varepsilon^{2}\right) \sin \theta \cos \theta}{1-\left(2 \varepsilon-\varepsilon^{2}\right) \cos ^{2} \theta}\right)^{2}+r^{2}\right]^{1 / 2},
$$

with 
$r(\theta)=\sqrt{\frac{a^{2} b^{2}}{a^{2} \sin ^{2} \theta+b^{2} \cos ^{2} \theta}}$.

The tangential velocity is simply given by $\mathrm{V}_{\mathrm{T}}=\Delta \mathrm{s} / \Delta \mathrm{t}$ and the integral (1) was numerically calculated for each tie-point. This method gives essentially the same result as that determined by measuring $\mathrm{V}_{\mathrm{T}}$ from the radial and orthogonal components of the velocity (polar coordinates) of the tracers. In Table 2 we give the positions and velocity of the most reliable tracers of the flow shown in figure 1 . Some other features where traced just outside the vortex, for example in the stagnant points at the vertex of the semi-major axis. On the average we find that $\mathrm{V}_{\mathrm{T}}=45 \pm 11 \mathrm{~ms}^{-1}$ at a distance $\mathrm{r}=2400$ $\mathrm{km}$. A fit to the radial dependence of the tangential velocity (assuming no polar angle dependence) gives:

$V_{T}=-1021+0.88 r-0.00018 r^{2}$,

for $\mathrm{r}$ given in $\mathrm{km}$ and $\mathrm{V}_{\mathrm{T}}$ in $\mathrm{ms}^{-1}$. The maximum speed from this parabolic fit is $52 \mathrm{~ms}^{-1}$.

\section{[Table 2]}

The mean vorticity, averaged over the area enclosed, is the circulation divided by the area enclosed (Hess, 1969)

$\varsigma=\frac{\oint \vec{V}_{T} \bullet d \vec{l}}{\pi a b} \approx \frac{V_{T} L_{e}}{\pi a b}$,

where $\mathrm{L}_{\mathrm{e}}$ is the length (perimeter) of the ellipse which can be approached using the Ramanujan formula by

$L_{e} \approx \pi \sqrt{2\left(a^{2}+b^{2}\right)}$. 
This gives an averaged vorticity $\zeta=4.0 \pm 1.5 \times 10^{-5} \mathrm{~s}^{-1}$, which is very close to the $3.3 \times 10^{-5} \mathrm{~s}^{-1}$ averaged ambient relative vorticity of the anticyclonic domain bounded in the North by an intense eastward jet stream at $47^{\circ} \mathrm{N}\left(\mathrm{u} \sim 150 \mathrm{~ms}^{-1}\right)$ and in the South by a weak westward jet at $39^{\circ} \mathrm{N}\left(\mathrm{u} \sim-20 \mathrm{~ms}^{-1}\right)$ (Figure 2) (Sánchez-Lavega et al., 2000). The averaged zonal flow velocity at the latitude of BS was $<\mathrm{u}\left(43.1^{\circ} \mathrm{N}\right)>=20.6 \pm 16.0 \mathrm{~ms}^{-1}$ (Sánchez-Lavega et al., 2000) and therefore, BS moved westward relative to the ambient flow with a relative speed $u_{B S}=-15.3 \mathrm{~ms}^{-1}$.

\section{[Figure 2]}

\section{The EPIC model atmosphere}

By working in isentropic coordinates and assuming an adiabatic atmosphere, the EPIC ("Explicit Planetary Isentropic-Coordinate") numerical model (Dowling et al. 1998) solves the fully non-linear hydrostatic equations for the horizontal velocity components. The model computes, among other variables, the potential vorticity $q$, which is a conservative quantity that can be used as an atmospheric tracer in an inviscid and adiabatic flow (Pedlosky, 1987).

As performed in our previous simulations in Jupiter (García-Melendo et al., 2005), the main free parameters of the simulated atmosphere model relate to the assumed separable meridional $u(\varphi)$ and vertical $u(z)$ wind profiles, and the vertical thermal profile. Only the meridional profile of the zonal wind at cloud top level is known in the simulated horizontal domain and is taken from Sánchez-Lavega et al. (2000). The vertical wind shear and thermal profile are mostly unknown quantities below the visible cloud deck and are the basic properties to be retrieved. 


\subsection{The thermal vertical profile}

The thermal profile is based on the radio occultation experiments performed by the Voyager mission (Lindal et al., 1985). Initially we extended it down to $\mathrm{P}=10 \mathrm{bar}$, assuming that in its upper part Saturn's atmosphere is a mixture of non-condensable gases $\mathrm{H}_{2}, \mathrm{He}$, and $\mathrm{CH}_{4}$, and the condensable species $\mathrm{NH}_{3}, \mathrm{NH}_{4} \mathrm{SH}$, and $\mathrm{H}_{2} \mathrm{O}$, after applying the thermo-chemical models proposed by Atreya et al. (1999). The thermal profile was corrected for a helium molar fraction of 0.13 according to the re-analysis performed by Conrath and Gautier (2000) of Voyager data and by assuming that hydrogen was in ortho-para equilibrium. This profile tends towards an adiabat near the 1 bar level, although it is slightly unstable $\left(N^{2}<0\right.$, being $N$ the Brunt-Väisälä frequency) for $P>400 \mathrm{mbar}$ (Figure 3). Nevertheless, the presence of vigorous convective phenomena and long lived vortices at mid latitudes in both hemispheres (SánchezLavega et al., 2003, 2004; Porco et al. 2005) suggests that statically stable as well as unstable areas must coexist in these bands of Saturn's atmosphere. We adopted the hypothesis that on the average the atmosphere in this region is statically stable since long-lived vortices form at all longitudes and that it becomes marginally unstable in short periods and small regions where convective storms form.

We adopted a simple vertical thermal structure by keeping the measured $N(P)$ profile in the upper part of the model atmosphere and making it constant below a fixed pressure value. To make the thermal profile continuous we adopted the approach used by LeBeau and Dowling (1998) in their simulations of Neptune's Dark Spot. According to their derivation and notation, assuming uniform stratification $\left(N^{2}=N_{0}^{2}=\right.$ constant $)$, an ideal gas behaviour, and by making use of the hydrostatic approximation, the $T(P)$ profile writes for $P \geq P_{0}$ 


$$
T(P)=\frac{\left(\frac{P}{P_{0}}\right)^{\kappa} T_{N} T_{0}}{T_{N}+\left[\left(\frac{P}{P_{0}}\right)^{\kappa}-1\right] T_{0}}
$$

Here $\kappa$ is the ratio of the gas constant to the specific heat at constant pressure and $T_{N} \equiv$ $\kappa g^{2} / N_{0}^{2} R_{\text {gas }}$ is fixed by the chosen value of both $N_{0}^{2}$ and the gas mixture, and governs the slope of $T(P)$. The pair $\left(P_{0}, T_{0}\right)$ is selected to assure temperature continuity between the stratospheric observed $T(P)$ profile and the constant $N(P)$ region bellow. Although for a given $T_{N}$ the pair $\left(P_{0}, T_{0}\right)$ can be arbitrarily chosen, if we want a reasonable match between our model $T(P)$ and the extended Saturn's profile, we may think of preserving the temperature minimum (the tropopause), and avoiding an excessive departure of our model $T(P)$ from the extended Saturn's profile. These two conditions restrict the $N_{0}{ }^{2}$ values that can be used. In our simulations we tested the two values of $N_{0}^{2}=0.03 \times 10^{-4} \mathrm{~s}^{-}$ 2 and $0.1 \times 10^{-4} \mathrm{~s}^{-2}$. The resulting $T(P)$ and $N^{2}(P)$ profiles are depicted in Figure 3.

\section{[Figure 3]}

\subsection{The wind profile}

We use the Voyager meridional wind profile at cloud top level as measured by Sánchez-Lavega et al. (2000) in our EPIC simulations. BS is located in the anticyclonic, south side, $47^{\circ} \mathrm{N}$ jet stream. Figure 2 depicts the part of the jet stream used in the simulations including its uncertainty due to navigation and measurement errors.

To deal with the vertical behaviour of the wind profile, we assumed that $U(\varphi, P)$ was the product of separate functions of latitude $\varphi$ and pressure $P$. This is in 
agreement with observed profiles in the upper troposphere and stratospheric thermal winds (Flasar et al., 2005) and it constitutes a convenient approximation to the unknown lower tropospheric winds. Therefore we use

$U(\varphi, P)=u_{h}(\varphi) u_{v}(P)$

where $u_{h}(\varphi)$ is the zonal wind profile at the cloud level $P_{0}$ and $u_{v}(P)$ is a non dimensional function normalized at the cloud level pressure $P_{0}\left(P_{0} \sim 500 \mathrm{mbar}, u_{v}\left(P_{0}\right)=\right.$ 1), which modulates the amplitude of $u_{h}(\varphi)$. As in García-Melendo et al. (2005), we adopted a piecewise structure for $u_{v}(P)$ in two linear segments (see Figure 4). For $P<$ $P_{0}$, and assuming an $m_{l}$ e-folding vertical scale height, the expression for $u_{v}(P)$ is

$u_{v}(P)=\left(1+m_{1} \ln \left(\frac{P}{P_{0}}\right)\right)$.

\section{[Figure 4]}

Our models were run for two different values of $m_{l}$. Ingersoll et al. (1984) found an average value of $m_{1}=0.2$ for the wind decay above the cloud deck based on the results published by Conrath and Pirraglia (1983). More recent results based on Cassini observations suggest that at midlatitudes there is almost no wind decay for Saturn's atmospheric layers above the 500 mbar level (Flasar et al., 2005). Although these Cassini results are limited to the planet's southern hemisphere, we also adopted as a second test value $m_{l}=0$. 
We have no information on the vertical wind shear below the ammonia upper cloud limit. Here is where we introduced our two-piece linear segment model to test different wind shear tendencies. For $P>P_{0}$, we extended this profile to a level $P_{1}$ where we switched to slope $m_{2}$, a parameter which could take positive, zero, or negative values. Below $P_{l}$ (higher pressure levels), we adopt the following expression for $u_{v}(P)$ :

$$
\begin{aligned}
& u_{v}(P)=\left(u_{1}+m_{2} \ln \left(\frac{P}{P_{1}}\right)\right) \\
& u_{1}=1+m_{1} \ln \left(P_{1} / P_{0}\right)
\end{aligned}
$$

Therefore, $m_{1}, N, P_{1}$ and $m_{2}$ are the basic atmospheric parameters to be retrieved from our simulations, although, $m_{1}$ and $N$ are somewhat constrained by the observations.

\subsection{Simulation domain}

Simulations were carried out in a channel spanning $60^{\circ}$ in longitude by $15^{\circ}$ in latitude with a grid of $256 \times 64$ points (in some cases $128 \times 32$, which represents the minimum number of points required to reproduce the vortex behaviour) for the horizontal domain. These values assured an equal latitudinal and longitudinal resolution of $0.24^{\circ}$ per grid point, or equivalently $235 \mathrm{~km}$ (respectively $0.47^{\circ}$ or $470 \mathrm{~km}$ ). The channel covered the planetographic latitude interval from $36^{\circ} \mathrm{N}$ to $51^{\circ} \mathrm{N}$. Vortex pair interactions were simulated at a grid resolution of $0.24^{\circ}$ in the same horizontal domain. The used time step length $\Delta \mathrm{t}$ was of 60 seconds for the low resolution simulations and 30 seconds in the high resolution experiments, well below the average Courant number limits of 100 and 55 seconds respectively. The vertical domain extends from $\mathrm{P}_{\text {top }}=10$ mbar to $\mathrm{P}_{\text {bottom }}=10$ bar and was divided in eight layers as described in García-Melendo 
et al. (2005). In this case, and as it can be appreciated in Figure 3, the bottom layer is an abysm with almost neutral stability.

\subsection{Single vortex simulations}

In the first set of simulations aimed to test the effects of the vertical wind shear and thermal profile on the vortex drift velocity and lifetime, we introduced a single vortex in the simulation domain. After specifying the vortex tangential velocity, its position, and its horizontal and vertical extension, EPIC introduces a geostrophically balanced vortex by modifying the initial $(t=0)$ Montgomery potential $M=C_{p} T+g z$, which appears as the forcing term in the isentropic-coordinate non-linear equations (Dowling et al., 1998). Afterwards, it is let to evolve freely (see Morales-Juberías et al., 2003). The vortex was introduced at latitude $43^{\circ} \mathrm{N}$ with initial tangential winds of 50 and $100 \mathrm{~ms}^{-1}$. Although this last value for $V_{T}$ is almost as double as the one measured from Voyager images, we wanted to make sure that the simulated vortex, which slowly damps out as the simulation goes on, acquired tangential velocities similar to the measured $V_{T}$ by the time it reached half of its lifetime span. In this way we also tested different values for the "hyperviscosity parameter" (see Table 3 and section 4.1). It was given the adequate initial size parameters to mimic the actual measured dimensions during its mature stage, again taken at half lifetime. With almost no difference in the results, we introduced the vortex at two different levels $P_{0}=1$ bar and $P_{0}=1.5$ bar where the putative ammonia cloud forms. Our vortex has the form of a Gaussian perturbation with a limited vertical extent of a few vertical scale heights. It is, therefore, thin relative to its much larger horizontal dimensions. The vortex vertical extension under the cloud deck is unknown, but the ratio between the vertical and horizontal scales should be of the order of $\sim f /(2 N)$, where $f$ is the Coriolis parameter as a 
characteristic horizontal frequency (Conrath et al., 1981). For the different possible values of $N$ and the scale height $H$, this estimated ratio may take any value between 2.0 and 4.5 scale heights, so we made simulations using vertical extents going upward from 2 to 3.5 and downwards from 3.0 to 4.0 as a possible working scale heights above and below BS visible clouds.

Table 3 summarizes the range of values used for each parameter in the simulations.

\section{[Table 3]}

\section{Results}

The main goal of this set of experiments was to identify which set of values of the parameter space resulted in a vortex drift rate of $\sim 5 \mathrm{~ms}^{-1}$ with respect to System III, and if it was also possible to maintain stable vortices with longevities longer than 1 year when the parameter combinations yielded good drift rates. For the physical model, we tested all the combinations for $P_{0}, P_{1}, m_{1}, m_{2}$, and $N(z)$, in addition to other parameter combinations directly related to the numerical computations as listed in Table 3.

\subsection{Drift velocities and vortex longevity}

Vortex drift velocities turned out to be very sensitive to the different combinations of $m_{1}, m_{2}$, and $P_{1}$. Our simulations suggested that drift rates could serve as a discriminator among the different model atmospheres. Figure 5 shows the resulting drift velocities for the low resolution simulations ( $128 \times 32$ grid point domain), $c_{u p}=2.5, c_{d o w n}=3.5$, and a hyperviscosity of $5.5 \times 10^{27} \mathrm{~m}^{6} \mathrm{~s}^{-1}$. The horizontal line marks the experimental drift rate 
for BS1. It can be seen that we obtain drift values close to $+5 \mathrm{~ms}^{-1}$ for $m_{2}$ around -0.2 , and that drift is a steep function of the vertical shear governed by $m_{2}$. For example, Figure 5 shows that and increment, positive or negative, of 0.2 around $m_{2}=-0.2$, introduces velocity variations of $\pm 15 \mathrm{~ms}^{-1}$. Positive values for the vertical wind shear below $P_{0}$ seem to be completely incompatible with our observations as they turn out into too high velocities. Panel A of Figure 2 displays the part of Saturn's zonal wind profile used in the simulations and, as solid dots, all the simulated drift velocities for BS1 represented in Figure 5.

\section{[Figure 5]}

The above mentioned results are of little value if we do not asses how errors in the local wind profile and simulation numerical parameters affect drift rates. Uncertainties in the zonal wind profile have two distinct sources. One has its origin in navigation errors, and it is given by the uncertainty associated to the position of tracers in the original spacecraft images. In this case latitude errors are specially important. The other source arises directly from the scatter of velocity measurements. All these uncertainties can be expressed as a velocity error bound about the measured nominal wind profile (SánchezLavega et al. 2000). We tested the influence of profile uncertainties in our numerical model by substituting the nominal profile by its upper and lower error limits. Panel B in Figure 2 shows the results. The solid dot is the drift velocity for $m_{1}=0.0, m_{2}=-0.2$, $P_{0}=500 \mathrm{mbar}, P_{I}=1000 \mathrm{mbar}, N^{2}=0.1 \times 10^{-4} \mathrm{~s}^{-2}$, a $128 \times 32$ point-grid, and the nominal wind profile. The light grey dots mark, respectively, the upper and lower new velocities when the wind profile upper and lower envelope limits are used. Maximum drift velocities variations accounted for 3 to $5 \mathrm{~ms}^{-1}$, which, for most of our different model 
atmospheres, implied at most a $+/-0.1$ uncertainty for the $m_{2}$ parameter. Therefore, wind profile errors yielded drift velocity alterations below our numerical model resolution.

A second source of uncertainties is the effect of the grid resolution and hyperviscosity on drift rate and vortex longevity. We found that the drift velocity, for any particular model atmosphere, was almost no sensitive to these parameters. Hyperviscosity, a parameter introduced to avoid growing numerical instabilities, can damp vortices out too quickly if it has a too high value. It is also directly related to grid resolution, and it can easily be decreased with increasing grid resolution. High grid resolution not only represents with much better detail any simulated phenomenon, but also allows using lower hyperviscosity values. The price to pay is the need for enormously longer CPU time. For instance, doubling grid resolution from $128 \times 32$ to $256 \times 64$, means the need of almost an order of magnitude longer CPU time, which is absolutely prohibitive if we want to fully explore all the combinations of the model atmosphere parameters. We performed some high and low resolution simulations with the same atmospheric parameters, and look at the impact on drift velocity. If drift velocity was not affected then we would explore all the parameter space at lower resolution. That was the situation, drift velocities derived from high and low resolution simulation were virtually indistinguishable for the same model atmosphere, and it was not affected for different values of hyperviscosity either.

Finally, vortex drift velocity was also almost independent of the simulation time, but vortex longevity was affected by grid resolution. Vortices had longer lifetimes for high resolution and low hyperviscosity values. A way of measuring the vortex longevity is simply looking at the potential vorticity field to see when the vortex dissolves. Another 
way of measuring longevity is looking at how the vortex tangential velocity evolves with simulation time. As an example, Figure 6 shows the evolution of maximum vortex tangential velocity with simulation time for the parameter combination $m_{1}=0.0, m_{2}=-0.2$, $P_{I}=1000 \mathrm{mbar}$, and $N^{2}=0.1 \times 10^{-4} \mathrm{~s}^{-2}$ for two different grid resolutions. For the low resolution case (grey line), vortex tangential velocity slows down at the beginning after vortex introduction, reaches a stable tangential velocity and afterwards, during dissipation, it starts oscillating until the vortex dissipates in the environment atmosphere. In the case of higher resolution, the tangential velocity remains stable with a very slow damping for a longer period of time. Despite the alterations of tangential velocity in low resolution simulations, the drift rates remained completely stable during the whole vortex lifetime. Low resolution simulations also provide a lower limit to the vortex longevity for each tested atmosphere. As an example, Figure 7 shows the evolution of drift velocities for all the simulated values of $m_{2}$ for the case $m_{l}=0.0$, $P_{I}=1000 \mathrm{mbar}, N^{2}=0.1 \times 10^{-4} \mathrm{~s}^{-2}$ and a $128 \times 32$ grid. They remained stable within 2 to 3 $\mathrm{ms}^{-1}$ until vortices dissolved, which again is well below the model parameter resolution.

\section{[Figure 6]}

\section{[Figure 7]}

Drift velocities close to the experimental value must be associated to longevities above 1 year, a lower bound imposed by observations. Figure 8 shows the measured longevities for the different combinations of the parameter space for the low resolution simulations. If we take into account that higher resolution grids result in simulated vortices with longer longevities, Figure 8 represents a lower bound which indicates that long-lived vortices in our model atmosphere are compatible with the adequate drift 
velocity.

\section{[Figure 8]}

\subsection{Global single vortex test results}

We found model atmospheres where simulated vortices matched reasonably well the observed properties. In all cases the best results were obtained for negative values of $m_{2}$. Although it is difficult to asses which is the "best" atmosphere model there are some trends resulting from the simulations. First, it is not possible to obtain drift velocities of $5 \mathrm{~ms}^{-1}$ for $m_{1}=0.2$ and $\mathrm{N}^{2}=0.03 \times 10^{-4} \mathrm{~s}^{-2}$ and only marginally for $\mathrm{N}^{2}=0.1 \times 10^{-4} \mathrm{~s}^{-2}$. This, of course, does not invalidate atmospheres with vertical wind shear above the visible cloud deck, but the better results for $m_{l}=0$ are more consistent with the Cassini results reported by Flasar et al. (2005). Second, lower values of $P_{I}$ seem to favour vortices with a longer lifetime and good drift values. And third, vortices introduced in atmospheres with negative $m_{2}$ values show an aspect ratio between the north-south semi-axis closer to the measured values for BS, while for progressively larger positive values of $m_{2}$, the vortices tend to be more elongated.

\subsection{Latitude migration experiments}

Since the meridional size of the vortex is smaller than the latitudinal width of the band comprised between the westward jet at $39^{\circ} \mathrm{N}$ and the eastward jet at $47^{\circ} \mathrm{N}$, a latitude migration of BS was in principle possible, so we explore this possibility in our experiments. In the first simulation we introduced the anticyclone at $\varphi=45^{\circ} \mathrm{N}$ and in the second at $41^{\circ} \mathrm{N}$, i.e. displaced $\pm 2^{\circ}$ from its nominal latitude, using a model atmosphere 
with a high static stability $\left(N_{0}^{2}=0.1 \times 10^{-4} \mathrm{~s}^{-2}\right)$, and $m_{1}=0.0, m_{2}=-0.2$ and $P_{1}=500 \mathrm{mbar}$. Figure 9 shows that there is no evidence of a large migration for the two studied cases. The vortex introduced at $41^{\circ} \mathrm{N}$ just oscillated around this latitude. When introduced at $45^{\circ} \mathrm{N}$ it migrated about one degree to the south during the initial first days, staying afterwards about 1 degree to the north of the measured BS position.

\section{[Figure 9]}

\section{Vortex pair interactions}

There is still a lack of detailed observations about the dynamical processes that occur during vortex merging in Saturn. Smith et al. (1982) and Sromovsky et al. (1983) showed examples of mergers between anticyclones that occurred in the latitude band of BS during the Voyager encounters. Porco et al. (2005) observed in Saturn's southern hemisphere using Cassini low resolution images, how two vortices spiralled toward each other during the merging process that took place in about three to four days.

In this new set of experiments we simulated the interaction between two vortices at high resolution to perform two types of tests. In the first one, two identical vortices were introduced in the domain separated by a small distance in latitude, one of them at $43^{\circ} \mathrm{N}$, the other typically one degree northwards. They were initially separated in longitude by about 40 degrees to make sure that the interaction would only occur after the short initial period of geostrophic adjustment of the EPIC model. In the second experiment, one of the vortices is introduced at $43^{\circ} \mathrm{N}$ with the same parameters as in the single-vortex experiments, but the second vortex is smaller, typically with dimensions about two-thirds that of the big one. Again, they are placed with a small separation in latitude, but a large one in longitude. 
We chose the same model atmosphere used to perform the vortex migration experiments. When vortices settled after their introduction, they approached each other showing a centre-to-centre separation of 3 degrees. Figure 10 illustrates the results. The left column shows our simulations when both vortices are identical. During the interaction process, orbiting started to take place when both vortices were $\sim 5$ longitude degrees apart, they then quickly spiralled around each other in a symmetric merging to produce a single vortex in about three days, which agrees with the temporal scale observed for the southern hemisphere vortex mergers (Porco et al., 2005). The case of two vortices with different sizes is shown in the right column of Figure 9. In this case, the small vortex starts orbiting the big one when they are between 7 and 10 degrees apart in longitude, resulting in a mixing process that again, takes about three days. In this latter case it is interesting to note that the merging process is accompanied by the formation of potential vorticity filaments (i. e., fluid filaments) linking both spots. These experiments strongly suggest that orbiting is a phenomenon accompanying a two vortex merger, and that the time required for the two vortices to merge as well as the orbiting phenomena depend on the flow pattern and relative speed of the two vortices.

\section{[Figure 10]}

\section{Discussion}

Our measurements clearly indicate that the BS vortex is in geostrophic balance with Rossby number Ro $=\mathrm{V}_{\mathrm{T}} / \mathrm{f} \mathrm{L} \sim 0.05$, being the planetary vorticity $\mathrm{f}=2 \Omega \sin \varphi=$ $2.2 \times 10^{-4} \mathrm{~s}^{-1}$ at the BS latitude. The vortex size $\mathrm{L}$ is above the Rossby deformation radius $\mathrm{L}_{\mathrm{D}}>\mathrm{Nh} / \mathrm{f} \sim 2200 \mathrm{~km}$ when using for $\mathrm{h}$ a vortex vertical extent $\sim 4 \mathrm{H}=150 \mathrm{~km}$ and for $\mathrm{N}>3.2 \times 10^{-3} \mathrm{~s}^{-1}$ as obtained from our simulations (see section 3 ). Its meridional extent nearly fills the domain between opposed jet streams (see figure 2), being constrained to 
the North by the "ribbon wave" (Sromovsky et al., 1983). Accordingly, the Burger number $\left(\mathrm{L}_{\mathrm{D}} / \mathrm{L}\right)^{2} \sim 0.08$ suggests that the vortex is stable against its tendency to be broken apart vertically, i. e. the density stratification in the vertical is low enough for the flow to be dominated horizontally by the planetary rotation (see e. g. Ingersoll et al., 1984). This is consistent with its lifetime $>1$ year that is well above its characteristic dynamic time $\tau_{\mathrm{dyn}}=\mathrm{L} / \mathrm{V}_{\mathrm{T}}$ and destruction time by the shear wind $\tau_{\text {shear }}=1 /(\partial \mathrm{u} / \partial \mathrm{y}) \sim 1$ day.

In Figure 11 we show how the zonal flow behaves in terms of a simple barotropic instability criterion at the latitude of BS. The magnitude of interest $\beta$ $\partial^{2} \mathrm{u} / \partial \mathrm{y}^{2}$ changes sign close to the BS zonal wind domain so the necessary condition (Rayleigh - Kuo criterion) for the barotropic instability to occur is met (Holton, 1992). In the more realistic situation we have simulated with EPIC, the baroclinic term should be considered to study the flow stability. However we do not consider this analysis due to the sensibility of this term to the uncertainties we have in the retrieved values for $\mathrm{N}(\mathrm{z})$ and $\mathrm{u}(\mathrm{z})$ below clouds. However we can address how large could be the contribution of the vertical wind shear to the vorticity of BS which is $\sim(f / N)^{2}(\partial \mathrm{u} / \partial \mathrm{z}) \sim$ $10^{-6}-5 \times 10^{-7} \mathrm{~s}^{-1}$ (using from our simulations $\partial \mathrm{u} / \partial \mathrm{z} \sim 10^{-4} \mathrm{~s}^{-1}$ and a lower limit for $\mathrm{N}>$ $\left.3 \times 10^{-3}\right)$. This is low and below the contribution of the horizontal wind shear vorticity $\left(\partial \mathrm{u} / \partial \mathrm{y} \sim 3 \times 10^{-5} \mathrm{~s}^{-1}\right)$ and that of the planetary vorticity $\mathrm{f}$ to the averaged BS vorticity $\zeta=$ $4 \times 10^{-5} \mathrm{~s}^{-1}$.

\section{Conclusions}

Our suite of anticyclone simulations for the main atmospheric model parameters $N_{0}{ }^{2}$, $m_{1}, m_{2}$, and $P_{1}$, suggests that in order to obtain stable vortices with lifetimes long enough to at least match the one-year observed survival for $\mathrm{BS}$, and to get drift 
velocities close to $5 \mathrm{~ms}^{-1}$, winds must slightly decay with depth down to the 10 bar level at the BS latitude. A more stable thermal profile $\left(N_{0}^{2}=0.1 \times 10^{-4} \mathrm{~s}^{-2}\right.$ below $\left.\log (P) \sim-1.0\right)$ yields more stable vortices. Simulations seem also favour constant winds or nearly so $\left(m_{1} \cong 0.0\right)$ above the visible cloud deck $\left(P_{0} \cong 500 \mathrm{mbar}\right)$, a result which seems to be consistent with the reported Cassini measurements of the thermal winds by Flasar et al. (2005). Finally, the value of $P_{1}$ (the level for the assumed inflexion in the vertical wind profile) is constrained by the zonal velocity results (see Figure 5), and locates it between the 500 to 1000 mbar level.

The overall picture from our model simulations at the BS latitude, and within the coarseness of the values of the parameter space, can be summarized in the following way. Saturn's upper troposphere at mid-latitudes has almost no wind shear above the 1 bar level. Below that level there is a slight wind decay with a vertical wind shear $\partial u / \partial z$ $\sim 2$ to $6 \mathrm{~ms}^{-1}$ per scale height in a slightly statically stable environment at the BS latitude. We would like to emphasize that our inferences on $N(z)$ and $u(z)$ are obtained within the context of the EPIC model and of the reference atmosphere we have used. They obviously are not unique in view of the unknowns of Saturn's deep atmosphere and the range of free parameters used. However and as far as we know, they are the first simulations specifically performed on Saturn's vortices and should serve as a guide to constrain realistically the structure of the upper troposphere (in the layers of the ammonia and water cloud formation) from future Cassini ISS observations of vortices (cyclones and anticyclones) at other latitudes. Measurements of the local zonal wind profile in their locations and of the temperature profiles and thermal stability from CIRS, VIMS and radio occultation, will allow us to extend our model simulations and constrain the dynamical properties of the atmosphere at different latitudes over a broad range of altitudes. 


\section{Acknowledgements}

This work has been supported by the research projects AYA2003-03216, FEDER and Grupos UPV 15946/2004. RH acknowledges a "Ramón y Cajal" contract from MEC. We acknowledge the National Space Data Center through the World Data Center A for Rockets and Satellites for providing the Voyager images. The Voyager Experiment Team Leader was Dr. Bradford A. Smith. This research also made use of the computing facilities at CESCA in Barcelona with the help of the Ministerio de Educación y Ciencia. 


\section{References}

Atreya, S.K., Wong, M.H., Owen, T.C., Mahaffy, P.R., Niemann, H.B., de Pater, I.,

Drossart, P., Encrenaz, T., 1999, A comparison of the atmospheres of Jupiter and Saturn: deep atmospheric composition, cloud structure, vertical mixing, and origin, Planet. Space Sci. 47, 1243-1262.

Conrath, B.J., Flasar, F. M., Pirraglia, J.A., Gierasch, P. J., and Hunt, G. E., 1981. Termal structure and dynamics of the Jovian atmosphere 2. Visible cloud features, $J$. Geophys. Res. 86, 8769-8775.

Conrath, B.J., Pirraglia, J.A., 1983. Thermal structure of Saturn from Voyager infrared measurements - Implications for atmospheric dynamics, Icarus 53, 286-292.

Conrath, B.J., Gautier, D., 2000. Saturn Helium Abundance: A Reanalysis of Voyager Measurements, Icarus 144, 124-134.

Desh, M.D., Kaiser, M.L., 1981. Voyager measurement of the rotation period of Saturn's magnetic field, Geophys. Res. Lett. 8, 253-256.

Dowling, T.E., Fisher, A.S., Gierasch, P.J., Harrington, J., LeBeau, R.P., Santori, C.M., 1998. The Explicit Planetary Isentropic-Coordinate (EPIC) Atmospheric Model, Icarus 132, 221-238. 
Flasar, F.M., $\quad$ Achterberg, R.K., $\quad$ Conrath, B.J., $\quad$ Pearl, J.C., $\quad$ Bjoraker, G.L., Jennings, D.E., $\quad$ Romani, P.N., $\quad$ Simon-Miller, A.A., $\quad$ Kunde, V.G., $\quad$ Nixon, C.A., Bézard, B., Orton, G.S., Spilker, L.J., Spencer, J.R., Irwin, P.G.J., Teanby, N.A., Owen, T.C., Brasunas, J., Segura, M.,E. Carlson, R.C., Mamoutkine, A., Gierasch, P.J., Schinder, P.J., Showalter, M.R., Ferrari, C., Barucci, A., Courtin, R., Coustenis, A., Fouchet, T., Gautier, D., $\quad$ Lellouch, E., Marten, A., $\quad$ Prangé, R., Strobel, D. F., Calcutt, S. B., Read, P. L., Taylor, F. W., Bowles, N., Samuelson, R. E., Abbas, M.M., Raulin, F., Ade, P., Edgington, S., Pilorz, S., Wallis, B., Wishnow, E. H., 2005. Temperatures, Winds, and Composition in the Saturnian System, Science 307, 12471251.

García-Melendo, E., Sánchez-Lavega, A., Dowling, T.E., 2005. Jupiter's $24^{\circ} \mathrm{N}$ highest speed jet: Vertical structure deduced from nonlinear simulations of a large-amplitude natural disturbance. Icarus 176, 272-282.

Gurnett, D. A., Persoon, A. M., Kurth, W. S., Groene, J. B., Averkamp, T. F., Dougherty, M. K., and D. J. Southwood, 2007. The variable rotation period of the inner region of Saturn's plasma disk. Science, 316, 442-445.

Holton, J.R. 1992. An Introduction to Dynamical Meteorology. Academic Press, San Diego, (USA).

Ingersoll, A.P., Beebe, R. F., Conrath, B.J., Hunt, G.E., 1984. Structure and dynamics of Saturn's atmosphere, in Saturn, T. Gehrels and M. S. Matthews (eds.), University of Arizona press, Tucson, $195-238$. 
Ingersoll, A. P., Dowling, T. E., Gierasch, P. J., Orton, G. S., Read, P. L., Sánchez-Lavega, A., Showman, A. P., Simon-Miller, A. A., and Vasavada, A. R.. 2004 'Dynamics of Jupiter's Atmosphere' in “Jupiter: The Planet, Satellites \& Magnetosphere” (eds. F. Bagenal, W. McKinnon, T. Dowling), Cambridge University Press, Cambridge, MA., pp 105-128, Chap. 6.

Lebeau, R.P., Dowling, T.E., 1998. EPIC Simulations of Time-Dependent, ThreeDimensional Vortices with Application to Neptune's Great Dark Spot, Icarus 132, 239265.

Legarreta, J., Sánchez-Lavega, A., 2005. Jupiter’s Cyclones and Anticyclones Vorticity from Voyager and Galileo Images. Icarus 174, 178-191.

Lindal, G. F., Sweetnam, D. N., Eshleman, V. R., 1985, The atmosphere of Saturn: an analysis of the Voyager radio occultation measurements, Astron. J. 90, 1136-1146.

Morales-Juberías, R., Sánchez-Lavega, A., Dowling, T., 2003. EPIC simulations of the merger of Jupiter's White Ovals BE and FA: Altitude dependent behavior, Icarus 166, $63-74$.

Pedlosky, J., 1987, Geophysical fluid dynamics, second ed., Springer-Verlag, New York.

Porco, C., Baker, E., Barbara, J., Beurle, K., Brahic, A., Burns, J.A., Charnoz, S., Cooper, N., Dawson, D.D., Del Genio, A.D., Denk, T., Dones, L., Dyudina, U., Evans, M.W., Giese, B., Grazier, K., Helfenstein, P., Ingersoll, A.P., Jacobson, R.A., Johnson, 
T.V, McEwen, A., Murray, C.D., Neukum, V, Owen, W.M., Perry, J., Roatsch, T., Spitale, J., Squyres, S., Thomas, P., Tiscareno, M., Turtle, E., Vasavada, A. R., Veverka, J., Wagner, R., West, R., 2005. Cassini Imaging Science: Initial Results on Saturn's Atmosphere, Science 307, 1243-1247.

Sánchez-Lavega, A., Rojas, J.F., Acarreta, J.R., Lecacheux, J., Colas, F., Sada, P.V., 1997. New Observations and studies of Saturn's long-lived North Polar Spot, Icarus 128, 322-334.

Sánchez-Lavega, A., Rojas, J.F., Sada, P.V., 2000. Saturn's zonal winds at cloud level, Icarus 147, 405-420.

Sánchez-Lavega, A., Pérez-Hoyos, S., Rojas, J.F., Hueso, R., French, R. G., 2003. A strong decrease in Saturn's equatorial jet at cloud level, Nature 423, 623-625.

Sánchez-Lavega, A., Hueso, R., Pérez-Hoyos, S., Rojas, J.F., French, R. G., 2004. Saturn's Cloud Morphology and Zonal Winds Before the Cassini Encounter, Icarus 170, 519-523.

Smith, B.A., Briggs, G.A., Danielson, G.E., Cook II, A.F.,, Davies, M.E., Hunt, G.E., Masursky, H., Soderblom, L.A., Owen, T.C., Sagan, C., 1977. Voyager imaging experiment, Space Sci. Rev. 21, 103-127.

Smith, B.A., Soderblom, L., Beebe, R., Boyce, J., Briggs, G., Bunker, G., Collins, S.A., Hansen, C. J., Johnson, T.V., Mitchell, J. L., Terrile, R., Carr, M., Cook II, A.F., Cuzzi, 
J., Pollack, J.B., Danielson, G.E., Ingersoll, A.P., Davies, M.E., Hunt, G.E., Masursky, H., Shoemaker, E., Morrison, D., Owen, T., Sagan, C., Veverka, J., Strom, R., Suomi, V.E., 1981. Encounter with Saturn: Voyager 1 imaging science results, Science, 212, $163-190$.

Smith, B.A., Soderblom, L., Batson, R., Bridges, P., Inge, J., Masursky, H., Shoemaker, Beebe, E.R., Boyce, Briggs, J., Bunker, G.A., Collins, S.A., Hansen, C.J., Johnson, T.V., Mitchell, J.L., Terrile, R., Cook II, A.F., Cuzzi, J., Pollack, J.B., Danielson, G.E., Ingersoll, A.P., Davies, M.E., Hunt, G.E., Morrison, D., Owen, T., Sagan, C., Vveverka, J., Strom, R., Suomi, V.E., 1982. A new look at the Saturn System: the Voyager 2 images, Science 215, $504-537$.

Sromovsky, L.A., Rrevercomb, H.E., Krauss, R.J., Suomi, V.E., 1983. Voyager 2 Observations of Saturn's Northern Mid-latitude Cloud Features: Morphology, Motions and Evolution. J. Geophys. Res. 88, $8650-8666$.

Stone, P. H., 1976. The meteorology of the Jovian atmosphere, in Jupiter, T. Gehrels (ed.), University of Arizona press, Tucson, 586 - 618 .

Vasavada, A. R. and Showman, A. P., 2005. Jovian atmospheric dynamics: an update after Galileo and Cassini. Rep. Progr. Phys., 68, 1935-1996.

Vasavada, A. R., Hörst, S. M., Kennedy, M. R., Ingersoll, A. P., Porco, C. C., Del Genio, A. D., and West, R. A., 2006. Cassini imaging of Saturn: Southern Hemisphere winds and vortices, J. Geophys. Res., 111, E05004. 


\section{Figure captions}

\section{Figure 1.}

Green filtered images of the Brown Spot (BS) anticyclone obtained by Voyager 2 ISS on 23 August 1981 at 18:07:11 (left) and on 24 August 1981 at 04:21:35 (right). The cloud tracers of the vortex flow are identified by letters and their motions by wind vectors (see Table 2). Dashed wind vectors correspond to cloud features identified and measured in a different image pair. The vortex is centred at latitude $43.1^{\circ}$ North. Orientation is North up and East to the right.

\section{Figure 2}

Panels A and B show the zonal wind profile (grey band indicates the uncertainties) as measured by Sánchez-Lavega et al. (2000) that is used in the numerical simulations (nominal zonal wind profile). Solid dots in panel A mark the drift velocities of the simulated vortices for all the model atmosphere parameter combinations $\mathrm{m}_{1}, \mathrm{~m}_{2}, \mathrm{P}_{0}, \mathrm{P}_{1}$, and $\mathrm{N}^{2}$ (see Table 3). Only decreasing winds with depth resulted in drift velocities close to the experimentally measured values (horizontal line). The dark dot in panel B marks the drift velocity for $\mathrm{m}_{1}=0.0, \mathrm{~m}_{2}=-0.2, \mathrm{P}_{0}=500 \mathrm{mbar}, \mathrm{P}_{1}=1000 \mathrm{mbar}$, and $\mathrm{N}^{2}=0.1 \times 10^{-4} \mathrm{~s}^{-2}$ for the nominal zonal wind profile. When the nominal zonal profile is substituted by its upper and lower error limits, the resulting vortex drift velocities are marked by the light dots.

\section{Figure 3}


Upper panel. Saturn's thermal profiles between 1 mbar and 10 bar. The thick line represents the extended thermal profile determined by means of the radio occultation experiment performed by the Voyager spacecraft (Lindal et al., 1985) and the thin line represents the model thermal profile for $N_{0}^{2}=0.03 \times 10^{-4} \mathrm{~s}^{-2}$ below $\log (P) \sim-0.754$. The line with the thickness in between represents the thermal profile for $N_{0}^{2}=0.1 \times 10^{-4} \mathrm{~s}^{-2}$ below $\log (P)=-1$. Bottom panel. Vertical stability profiles in terms of the Brunt-Väisälä frequency $N^{2}$ corresponding to the previous line thickness code.

\section{Figure 4}

Vertical structure scheme of the zonal wind amplitude functions $u_{v}(P)$ used in our simulations. Two main cases are shown (grey $m_{1}=0.0$ and solid lines $m_{l}=0.2$ ), each one divided in the bottom part in the sub-cases corresponding to different values of the slope $m_{2}$ as specified in Table 3. $P_{0}$ is the cloud top level (500mbar) where $u_{v}\left(P_{0}\right)=1$.

\section{Figure 5.}

Simulated zonal vortex speeds for the different parameter combinations $\left(\mathrm{P}_{0}=500 \mathrm{mbar}\right)$ with a grid resolution of $0.47^{\circ}$. A) $\mathrm{m}_{1}=0.0, \mathrm{~N}^{2}=0.1 \times 10^{-4} \mathrm{~s}^{-2}$. B) $\mathrm{m}_{1}=0.0, \mathrm{~N}^{2}=0.03 \times 10^{-4} \mathrm{~s}^{-2}$. C) $\mathrm{m}_{1}=0.2, \mathrm{~N}^{2}=0.1 \times 10^{-4} \mathrm{~s}^{-2}$. D) $\mathrm{m}_{1}=0.2, \mathrm{~N}^{2}=0.03 \times 10^{-4} \mathrm{~s}^{-2}$. The horizontal line marks the measured BS1's drift speed.

\section{Figure 6.}

Evolution of the maximum tangential velocity as function of the simulated time and spatial resolution for a vortex with the parameter combination $\mathrm{m}_{1}=0.0, \mathrm{~m}_{2}=-0.2$, $\mathrm{P}_{1}=1000 \mathrm{mbar}$, and $\mathrm{N}^{2}=0.1 \times 10^{-4} \mathrm{~s}^{-2}$. The black and grey lines are the results for grid resolutions: 256x64 points (black line, $0.24^{\circ} \mathrm{pix}^{-1}$ ), 128x32 points (grey line $0.47^{\circ} \mathrm{pix}^{-1}$ ). 


\section{Figure 7.}

Vortex drift velocity for different values of the vertical wind shear parameter $m_{2}$ in the case: $\mathrm{m}_{1}=0.0, \mathrm{p}_{1}=1000 \mathrm{mbar}$, and $\mathrm{N}^{2}=0.1 \times 10^{-4} \mathrm{~s}^{-2}$. This graph is representative of other combinations of $\mathrm{m}_{1}, \mathrm{p}_{1}$, and $\mathrm{N}^{2}$, and shows that the drift velocity is a stable parameter along the life of the simulated vortex.

\section{Figure 8.}

Vortex longevity for different combinations of $P_{1}, m_{1}, m_{2}$, and $N_{0}{ }^{2}\left(P_{0}\right.$ is always fixed to 500 mbar), and a grid resolution of $128 \times 32$. The legend bar in the bottom part of the figure codes the maximum lifetime of the vortex in days, black is for a longevity up to 100 days, while white represent longevities longer than 900 days. For several cases of negative $m_{2}$, and specially for the case $N_{0}^{2}=0.03 \times 10^{-4} \mathrm{~s}^{-2}$, the resulting model atmosphere was unphysical, which means that isentropic surfaces crossed each other as a result of excessive vertical shear of the zonal wind to be consistent with hydrostatic and gradient-wind balance.

\section{Figure 9.}

Latitude position of two vortices, one introduced at $45^{\circ} \mathrm{N}$ (upper line) and the other at $41^{\circ} \mathrm{N}$ (bottom line) as a function of time for $N_{0}^{2}=0.1 \times 10^{-4} \mathrm{~s}^{-2}, m_{1}=0.0, m_{2}=-0.2$, and $P_{1}=500 \mathrm{mbar}$. The grey band represents de interval of latitude positions for BS measured by Sánchez-Lavega et al. (2000).

\section{Figure 10.}


Time sequence of the interaction and merging of two vortices represented by means of maps of the potential vorticity field. Left column sequence: Two vortices of similar size slightly separated in latitude. Right column sequence: Two vortices of different size (the smaller one is about 0.75 times the bigger one). On the right side of each frame the time simulation is written in days and hours.

\section{Figure 11}

Upper panel: Vorticity of the mean zonal flow (meridional profile) as derived from the observations (Sanchez-Laverga et al. 2000). Lower panel: representation of the difference between the planetary vorticity $\beta$ and $\partial^{2} u / \partial^{2} y$ as a function of latitude. The vertical grey line marks the position of BS1. The light grey ellipsoid represents the vortex BS-1 at the latitude scale. 
Table 1

Voyager 2 images selected to study the "Brown Spot" (BS) anticyclone

\begin{tabular}{|c|c|c|c|}
\hline$\underline{\text { PDS Volume }}$ & $\underline{\text { Image Number }}$ & $\underline{\text { Date }}$ & $\underline{\text { Time }}$ \\
\hline 4 & C4381308 & August 19, 1981 & $17: 24: 47$ \\
\hline 4 & C4382827 & August 20, 1981 & $05: 39: 59$ \\
\hline 4 & C4383930 & August 20, 1981 & $14: 30: 23$ \\
\hline 4 & C4385247 & August 20, 1981 & $01: 07: 59$ \\
\hline 4 & C4386551 & August 21, 1981 & $11: 35: 11$ \\
\hline 4 & C4387908 & August 21, 1981 & $22: 12: 47$ \\
\hline 5 & C4383401 & August 23, 1981 & $18: 07: 11$ \\
\hline 5 & C4384649 & August 24, 1981 & $04: 21: 35$ \\
\hline
\end{tabular}


Table 2

Tangential velocity of the flow around the "Brown Spot" (BS) anticyclone

\begin{tabular}{|c|c|c|c|}
\hline Feature & $\begin{array}{c}\text { Position angle } \\
<\theta>\end{array}$ & $\begin{array}{c}\text { Radial distance } \\
<r>\end{array}$ & $\begin{array}{c}\text { Tangential Velocity } \\
\qquad V_{T}\left(\mathrm{~ms}^{-1}\right)\end{array}$ \\
\hline A & 80.2 & 2227 & 62.6 \\
\hline B & 47.1 & 2583 & 41.2 \\
\hline $\mathrm{C}$ & 11.3 & 2266 & 55.9 \\
\hline $\mathrm{D}$ & 330.1 & 2632 & 44 \\
\hline $\mathrm{E}$ & 311.5 & 2323 & 38 \\
\hline $\mathrm{F}$ & 185.6 & 2204 & 14.5 \\
\hline $\mathrm{G}$ & 218.9 & 2518 & 59.2 \\
\hline $\mathrm{H}$ & 323.4 & 2258 & 53.8 \\
\hline I & 43.9 & 2153 & 33.4 \\
\hline $\mathrm{J}$ & 0.5 & 2800 & 31.3 \\
\hline $\mathrm{K}$ & 358 & 2076 & 30.4 \\
\hline $\bar{L}$ & 224.4 & 2212 & 52.6 \\
\hline $\mathrm{M}$ & 345 & 2670 & 30.4 \\
\hline
\end{tabular}


Table 3

Range of values of the parameters used in the simulations

\begin{tabular}{|c|c|}
\hline Parameter & Range of values \\
\hline $\mathrm{P}_{0}$ (mbar) & 500 \\
\hline $\mathrm{P}_{1}$ (mbar) & $500,1000,1500,2500$ \\
\hline $\mathrm{m}_{1}$ & $0.0,+0.2$ \\
\hline $\mathrm{m}_{2}$ & $-0.4,-0.3,-0.2,-0.1,0.0,+0.1,+0.2,+0.3$ \\
\hline Cup (scale heights) & $2.0,2.5,3.5$ \\
\hline Cdown (scale heights) & $3.0,3.5,4.0$ \\
\hline Vortex height (mbar) & 1000,1500 \\
\hline Initial $\mathrm{Vt}\left(\mathrm{ms}^{-1}\right)$ & 50,100 \\
\hline Vortex size $(a, b)$ & $6.5^{\circ} \times 3.7^{\circ}$ \\
\hline $\mathrm{N}^{2}(\mathrm{z})($ minimum $)\left(10^{-4} \mathrm{~s}^{-2}\right)$ & $0.03,0.1$ \\
\hline Domain & Long: $\left(-30^{\circ} \mathrm{a}+30^{\circ}\right)$, Lat $\left(+36^{\circ} \mathrm{a}+51^{\circ}\right)$ \\
\hline Number of layers & 8 \\
\hline $\mathrm{x}-\mathrm{y}$ resolution (points) & $128 \times 32,256 \times 64$ \\
\hline$v^{6}\left(10^{27} m^{6} s^{-1}\right)$ & $0.18,2.2,2.8,5.5,8.8$ \\
\hline$\Delta \mathrm{t}$ (seconds) & 30,60 \\
\hline
\end{tabular}




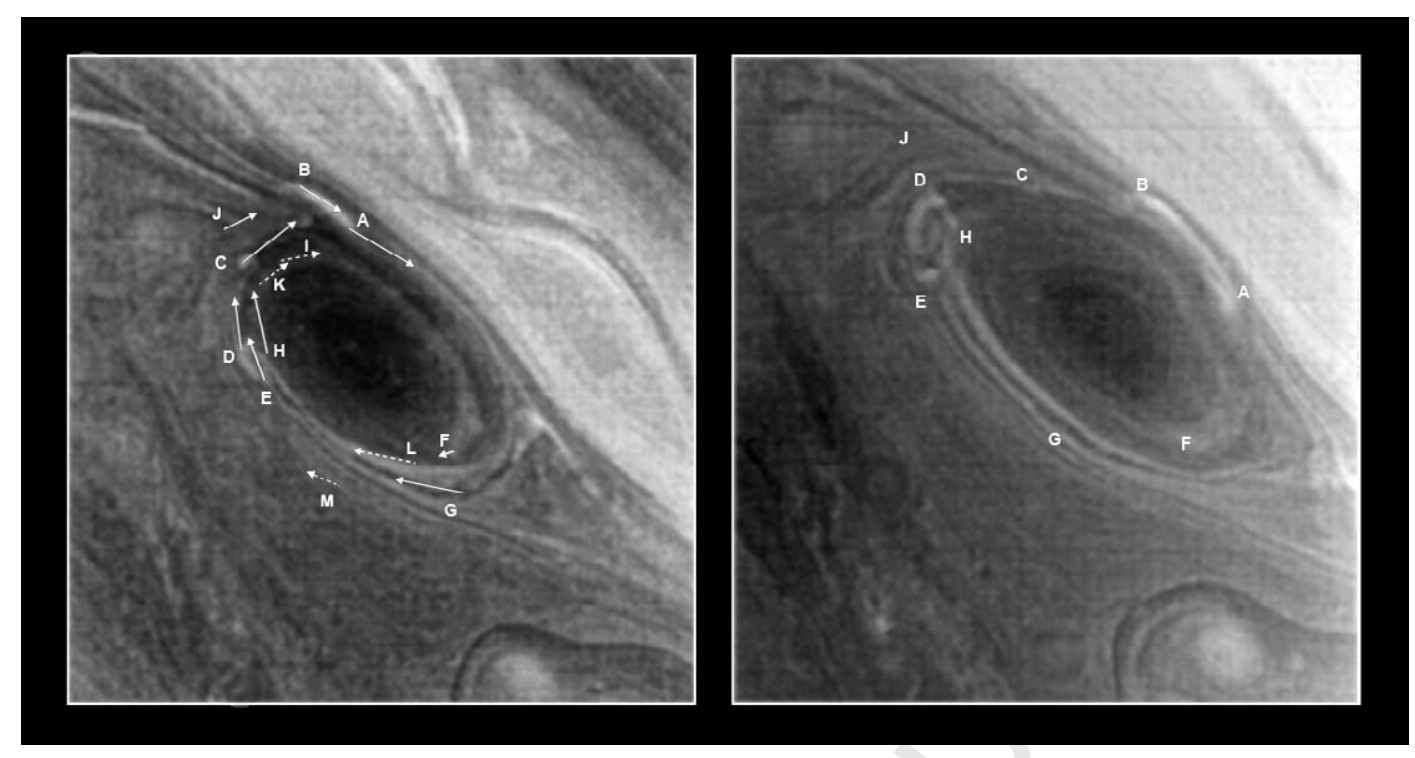

Figure 1 

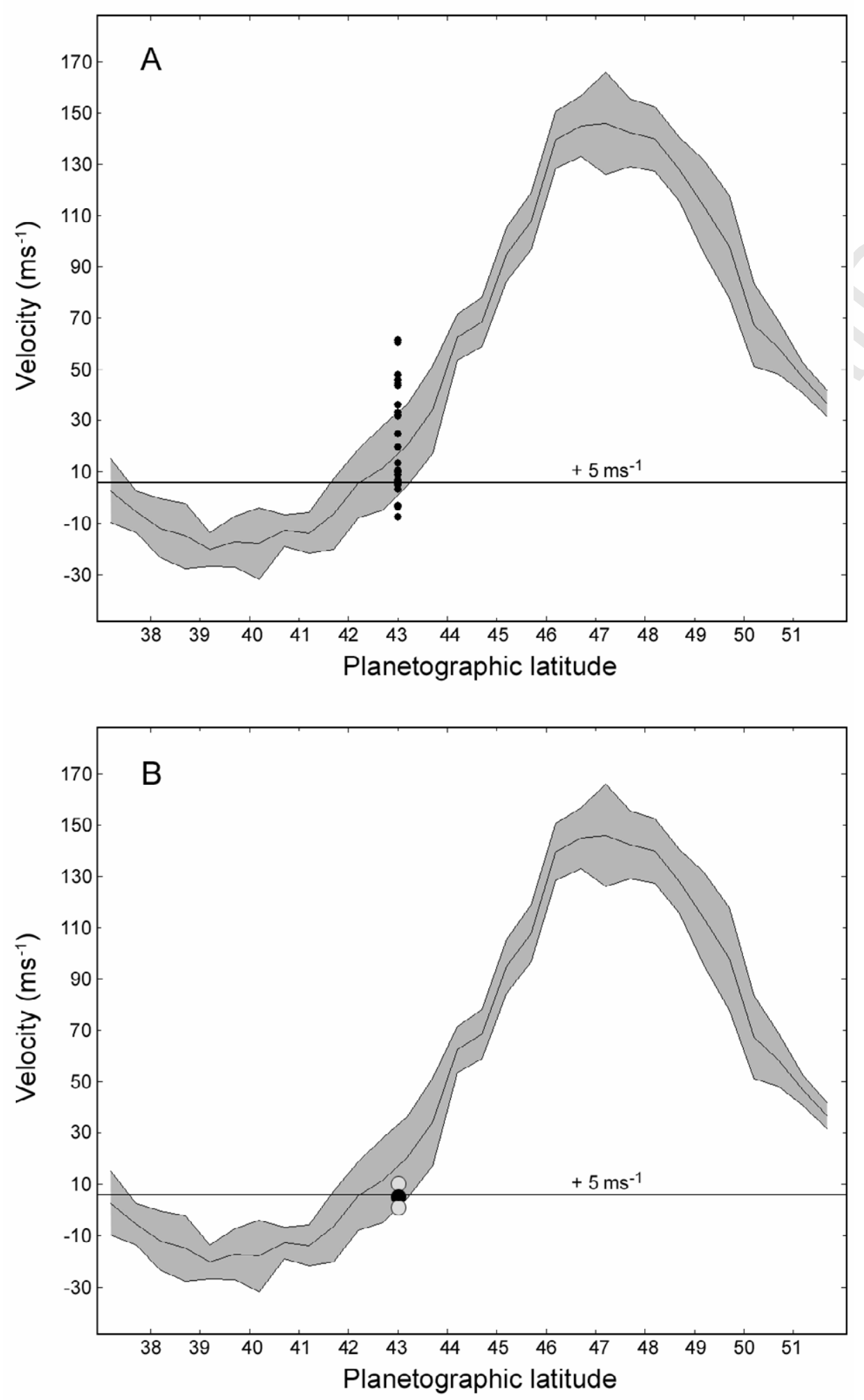

Figure 2 

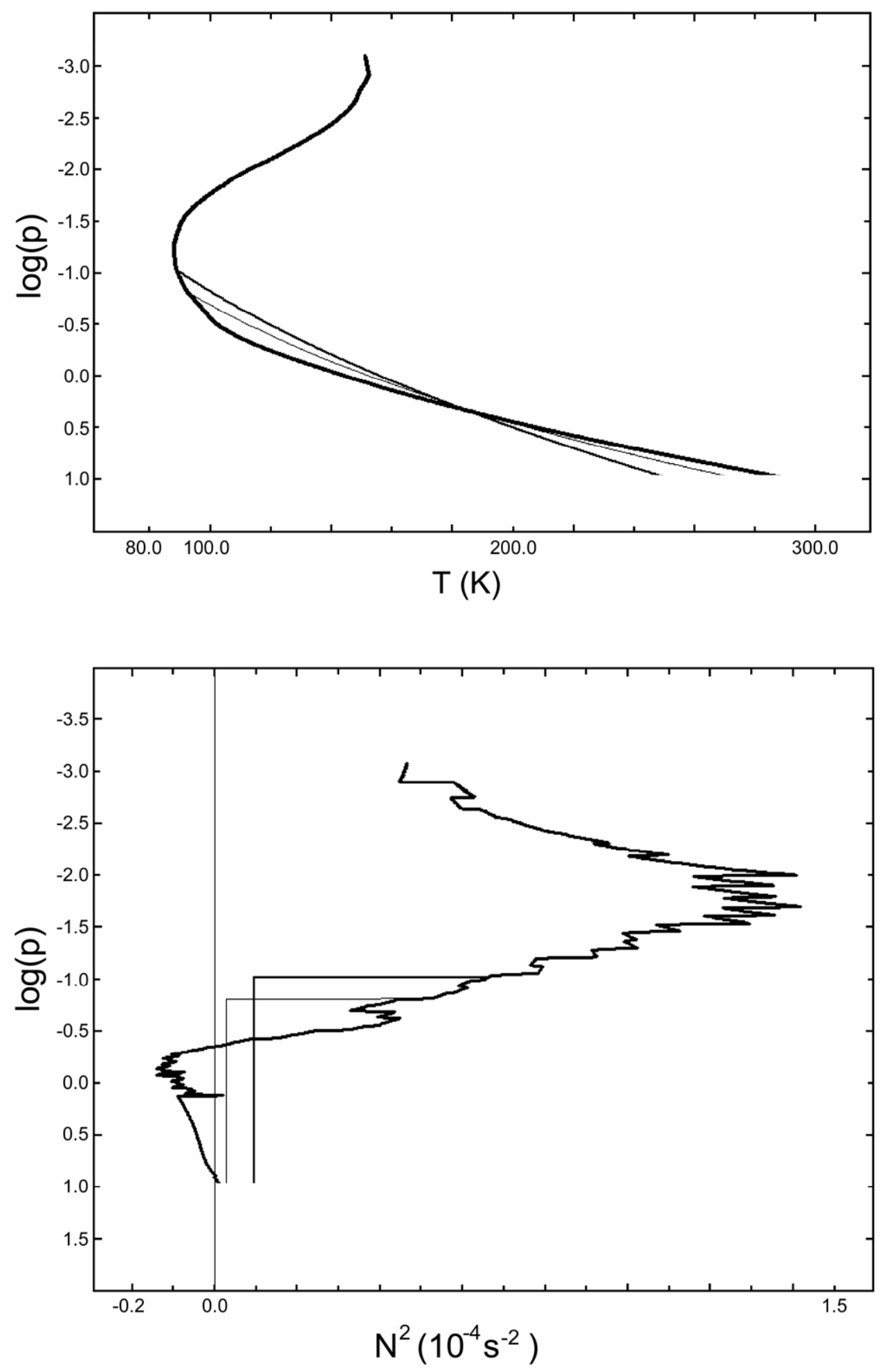

Figure 3 


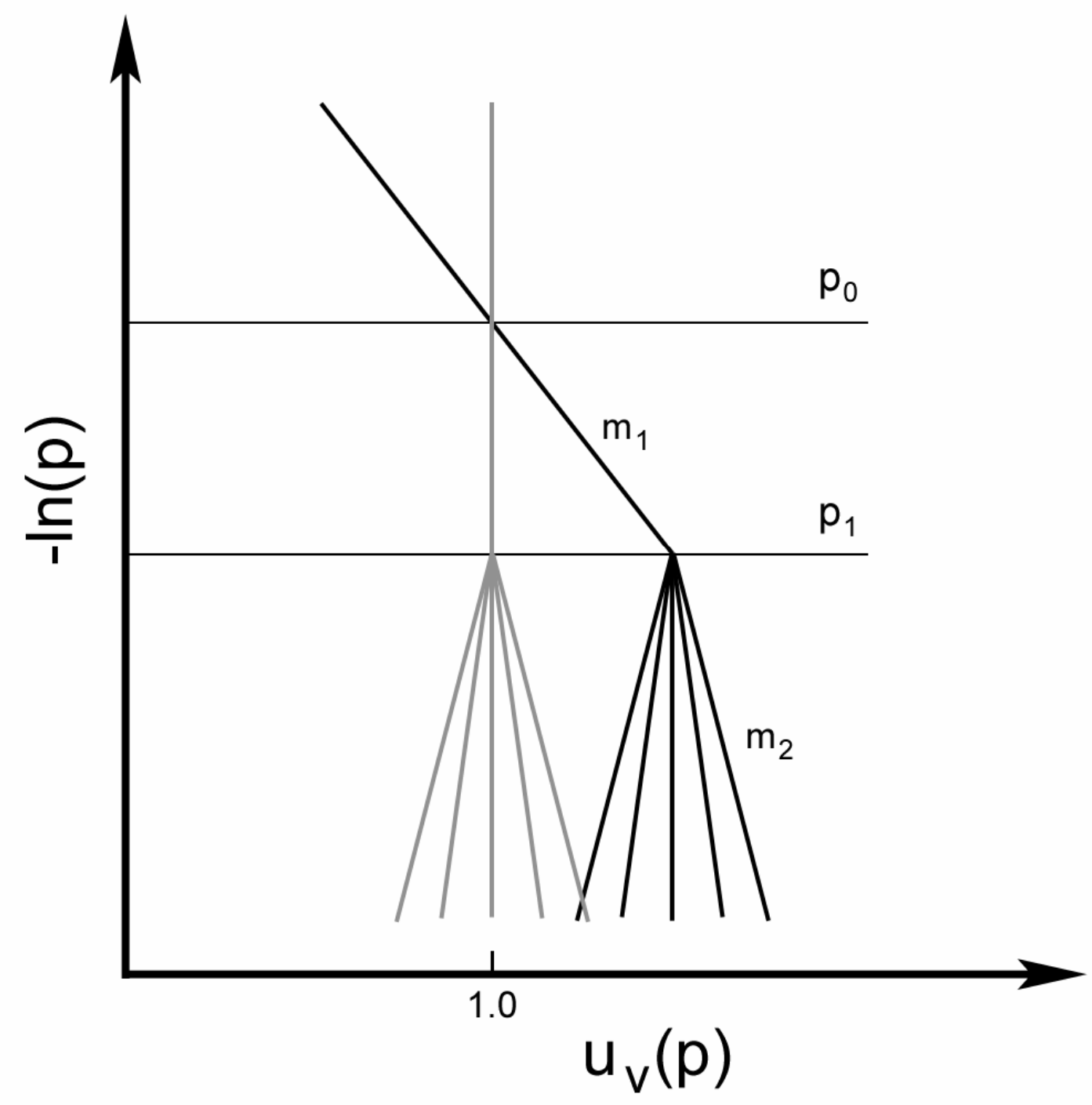

Figure 4 


\section{ACCEPTED MANUSCRIPT}
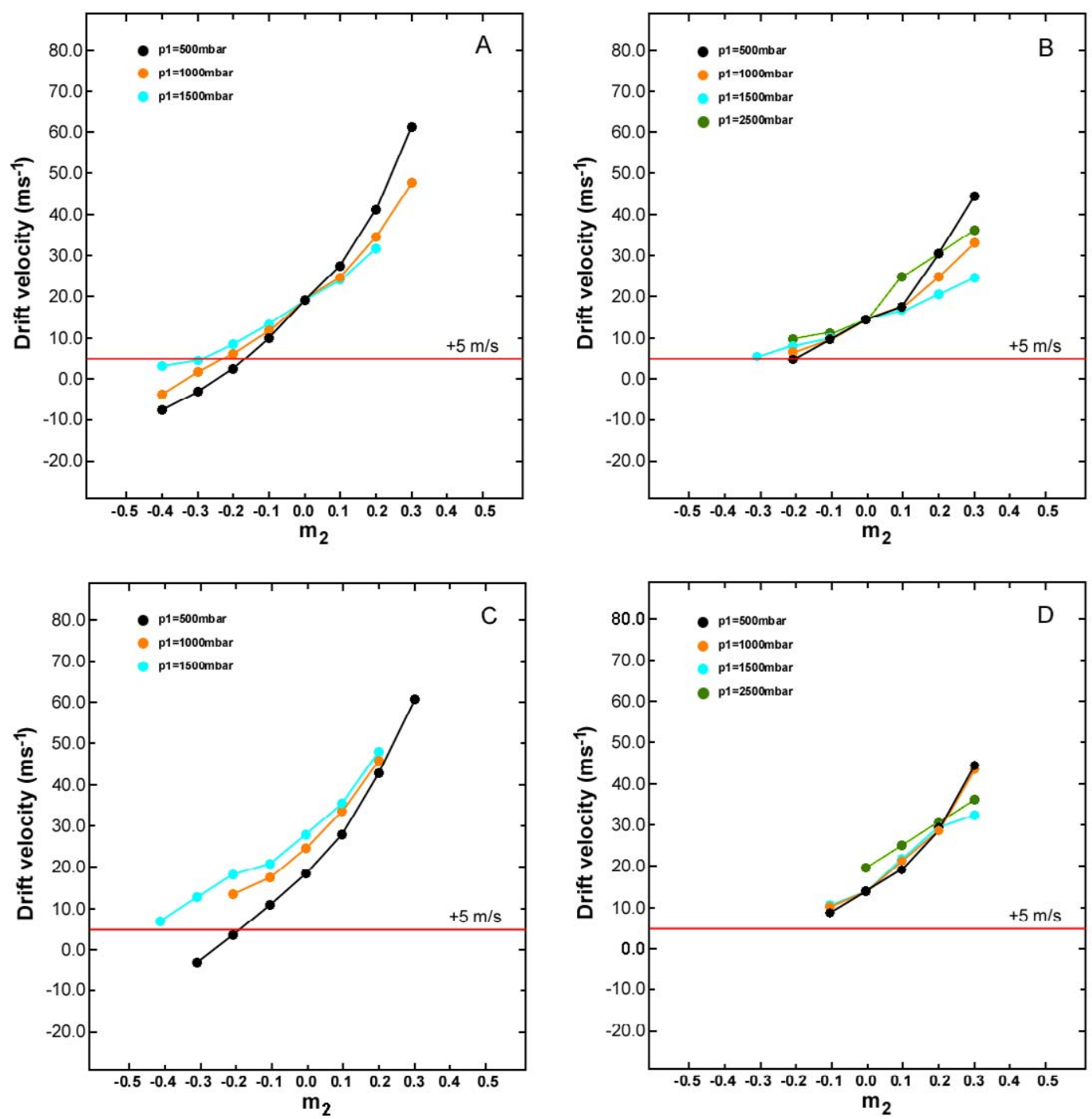

Figure 5 


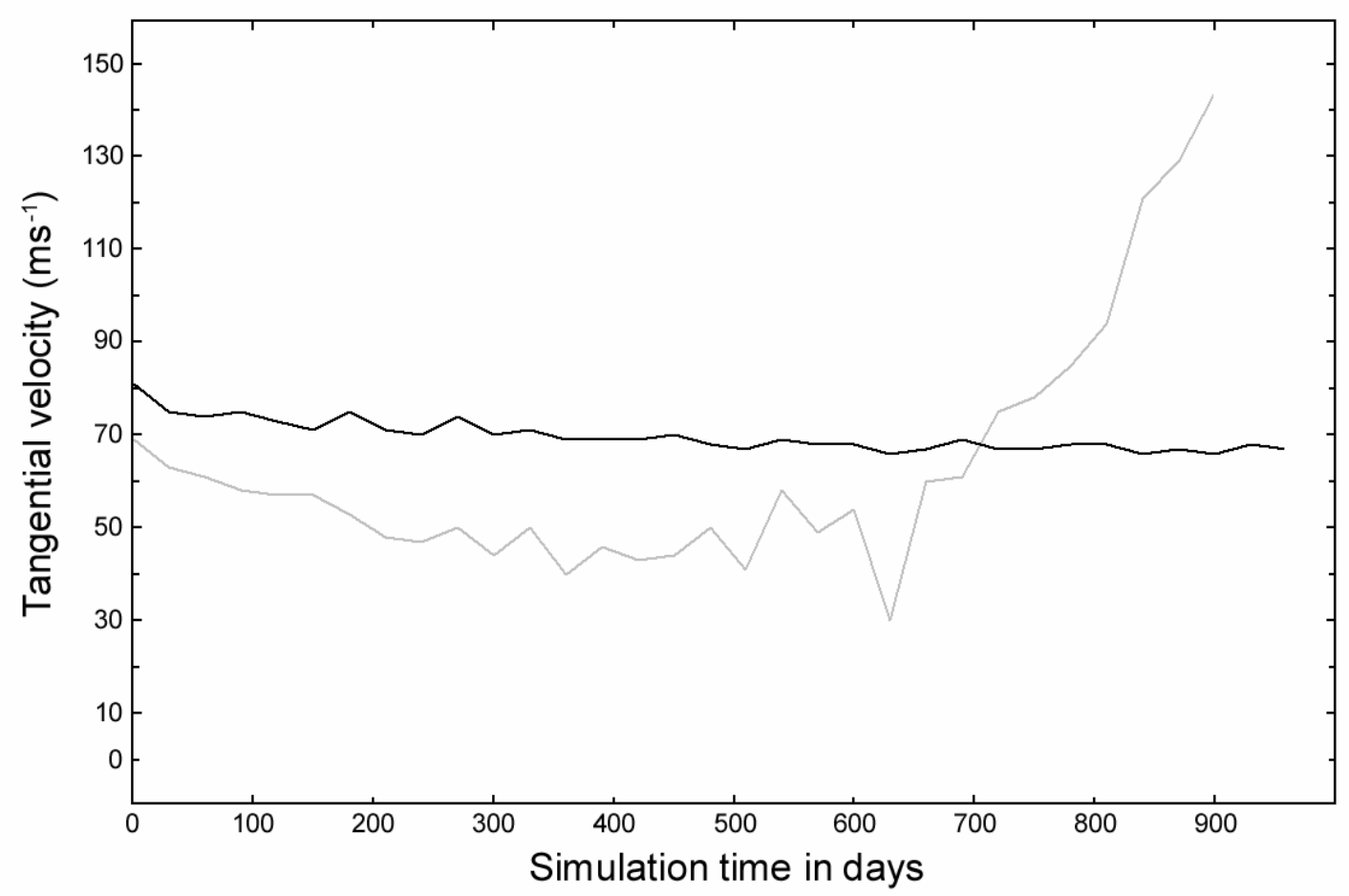

Figure 6 


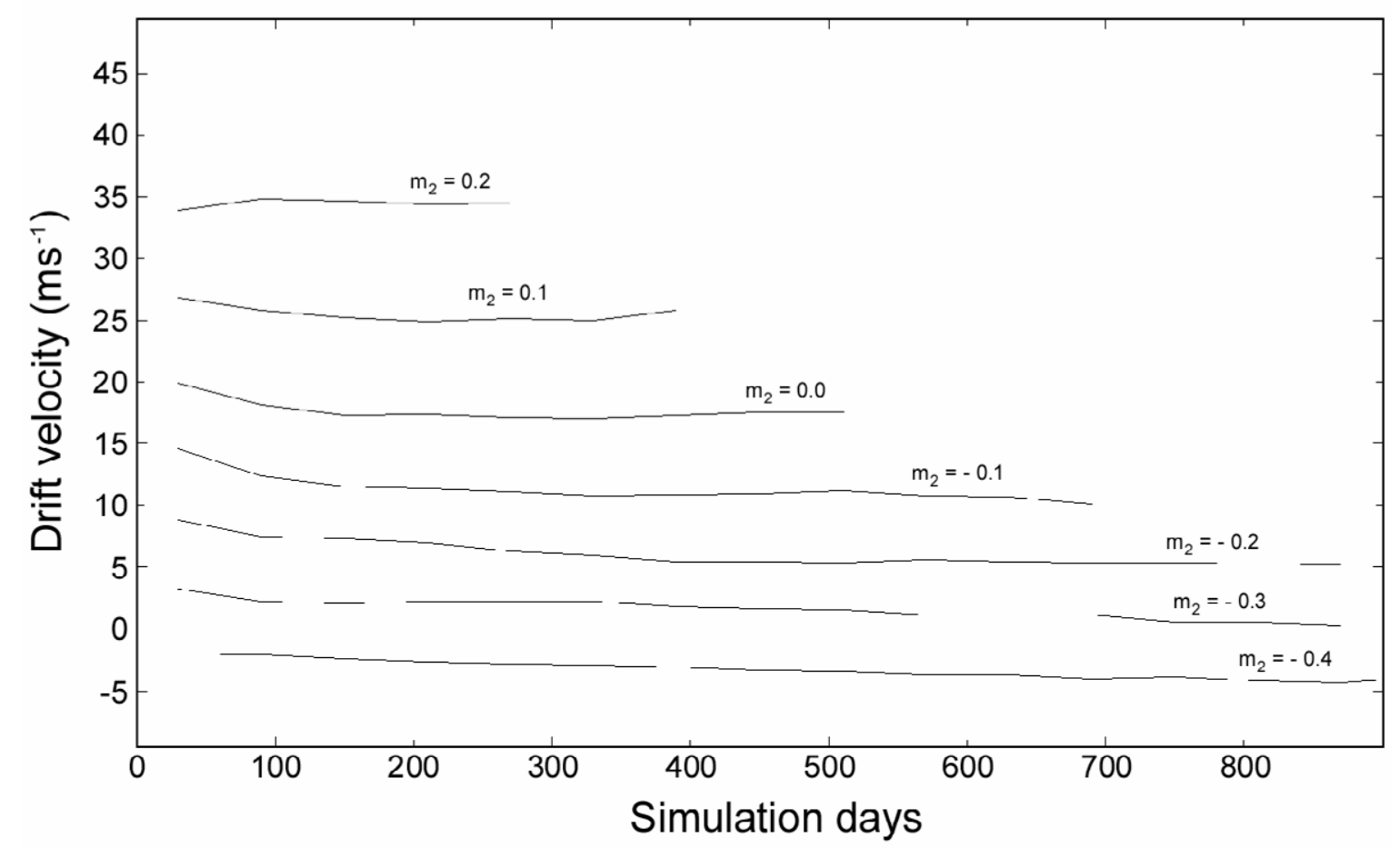

Figure 7 

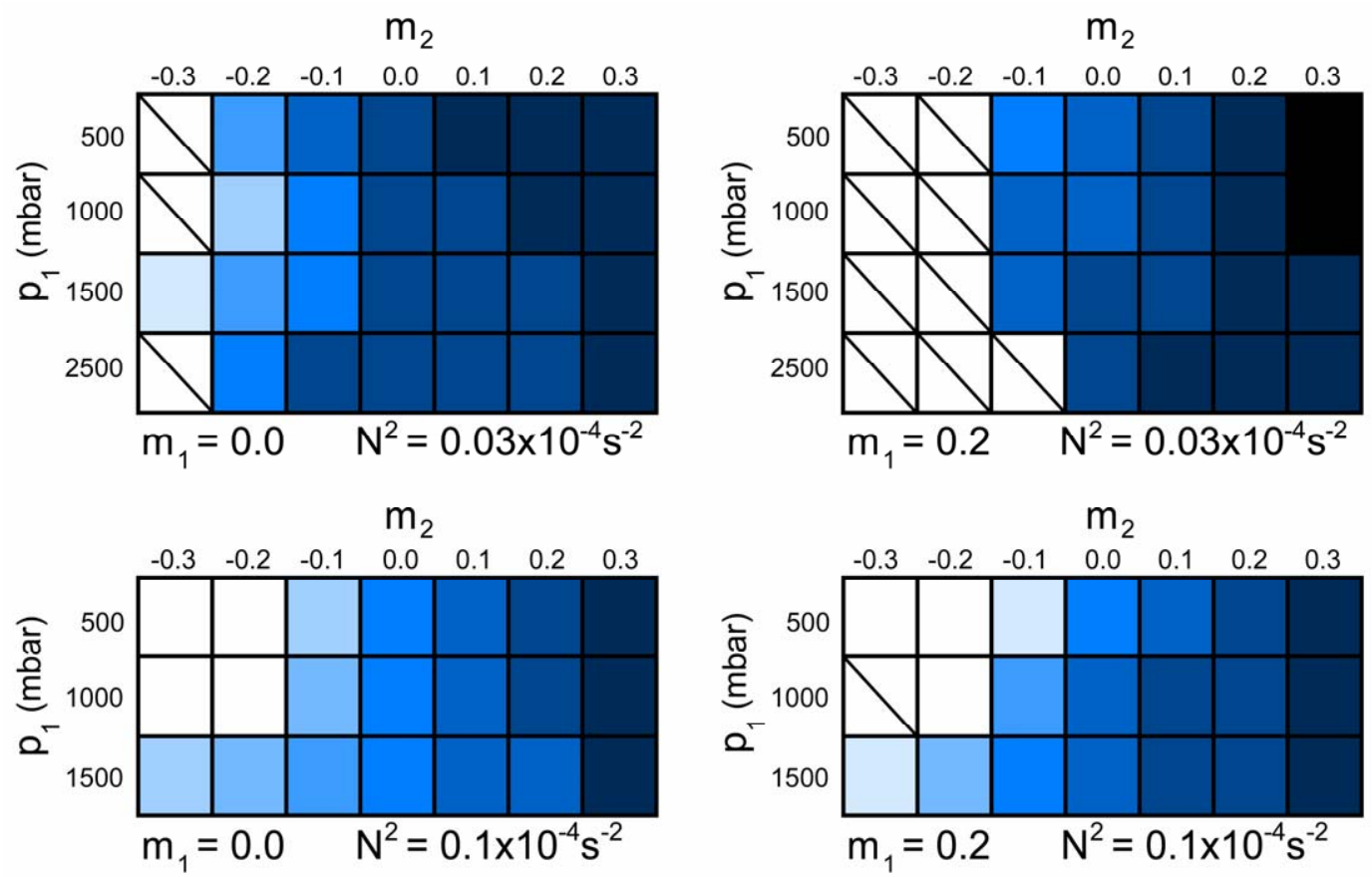

Lifetime in days

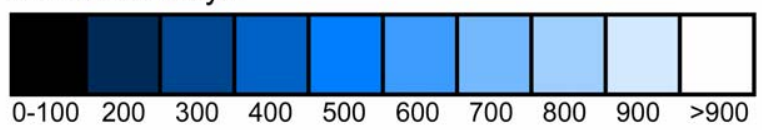

$\searrow$ Unphysical atmosphere

Figure 8 


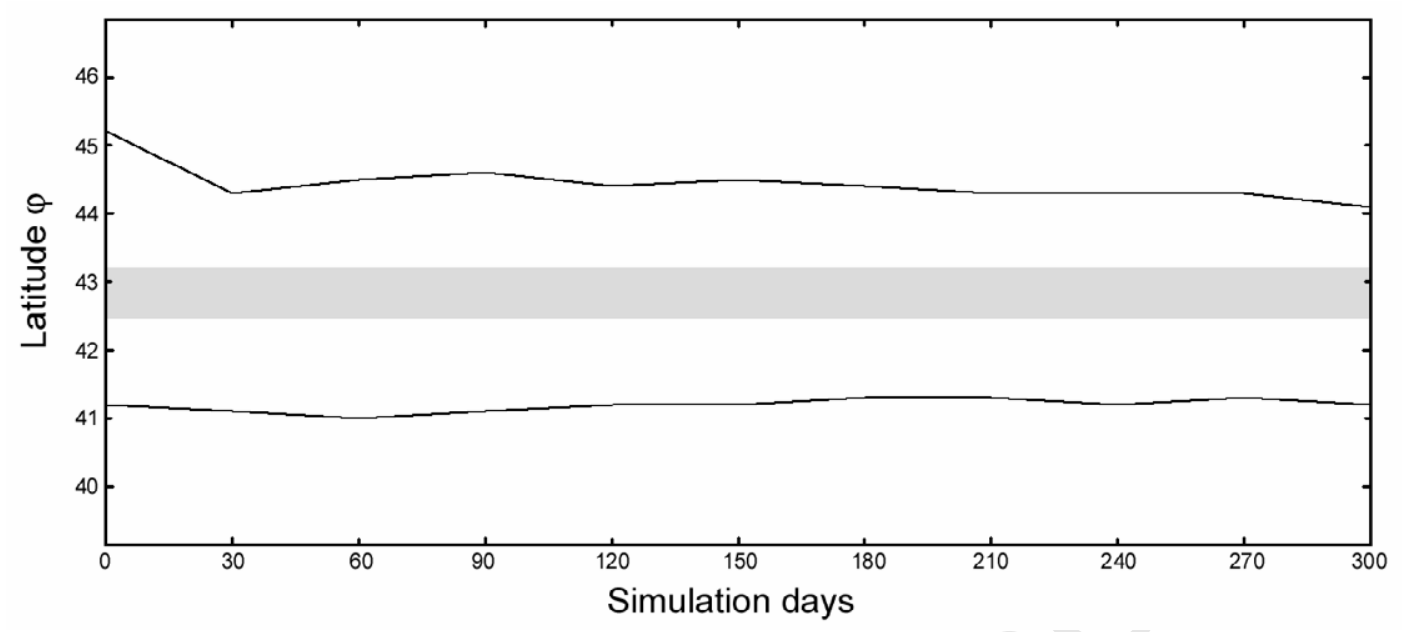

Figure 9. 

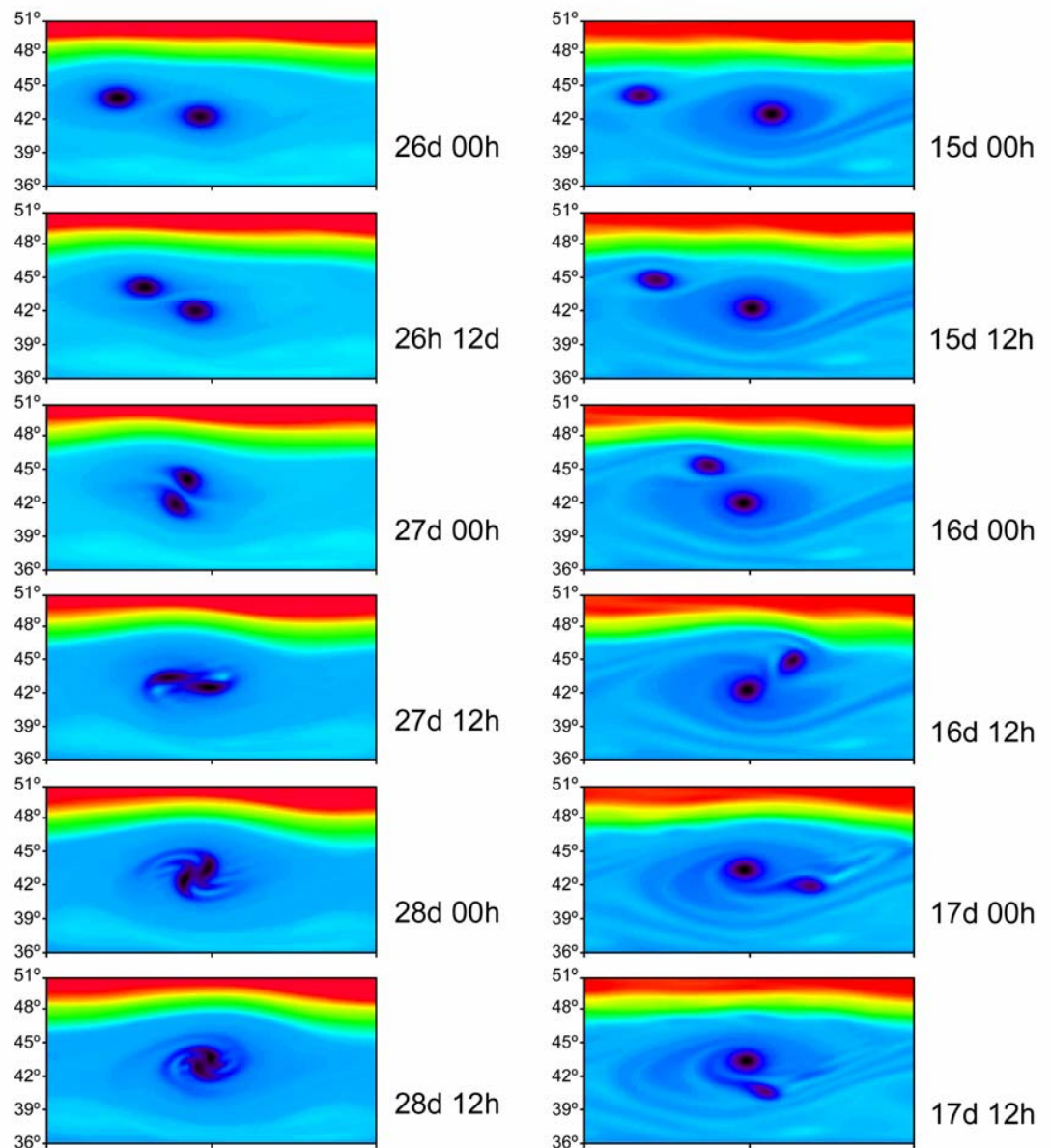

$17 \mathrm{~d} 00 \mathrm{~h}$

$28 d 12 h$
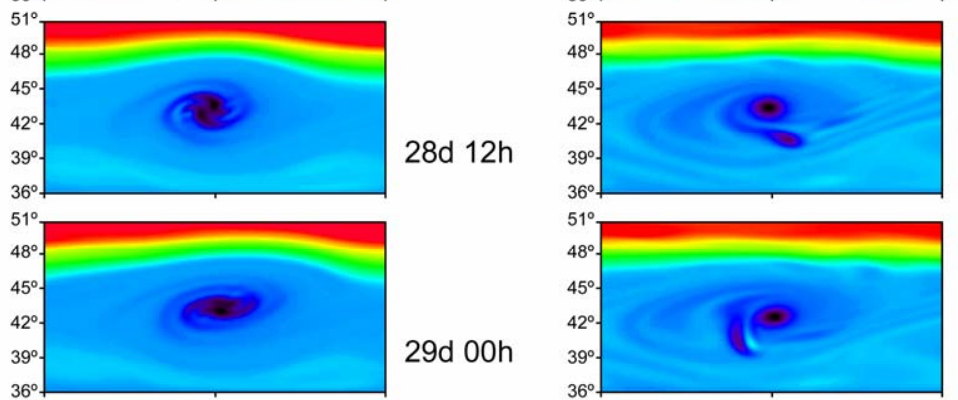

$17 \mathrm{~d} 12 \mathrm{~h}$
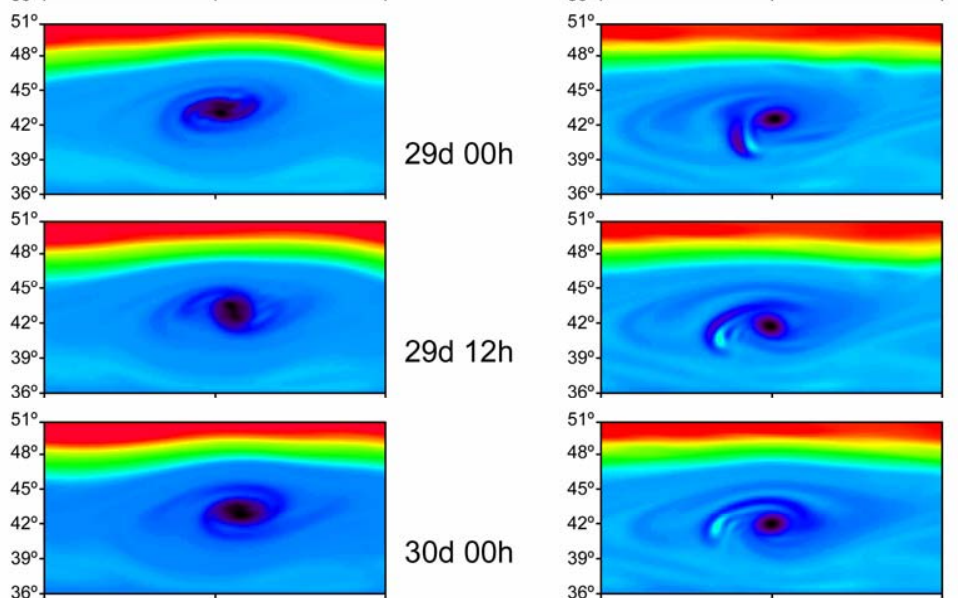

$18 \mathrm{~d} 00 \mathrm{~h}$

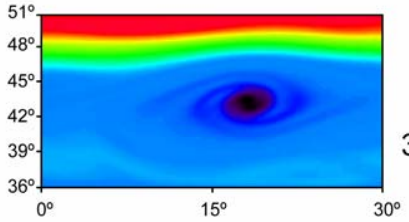

$30 \mathrm{~d} 12 \mathrm{~h}$

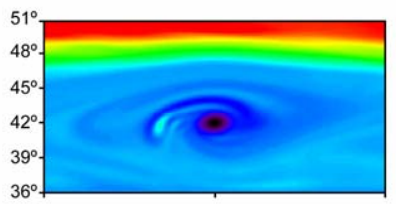

$18 d 12 h$

$19 \mathrm{~d} 00 \mathrm{~h}$

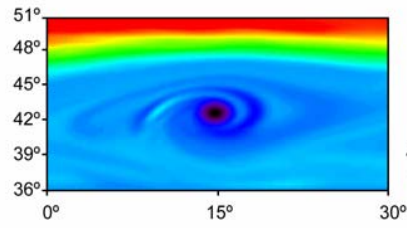

$19 \mathrm{~d} 12 \mathrm{~h}$

Figure 10 


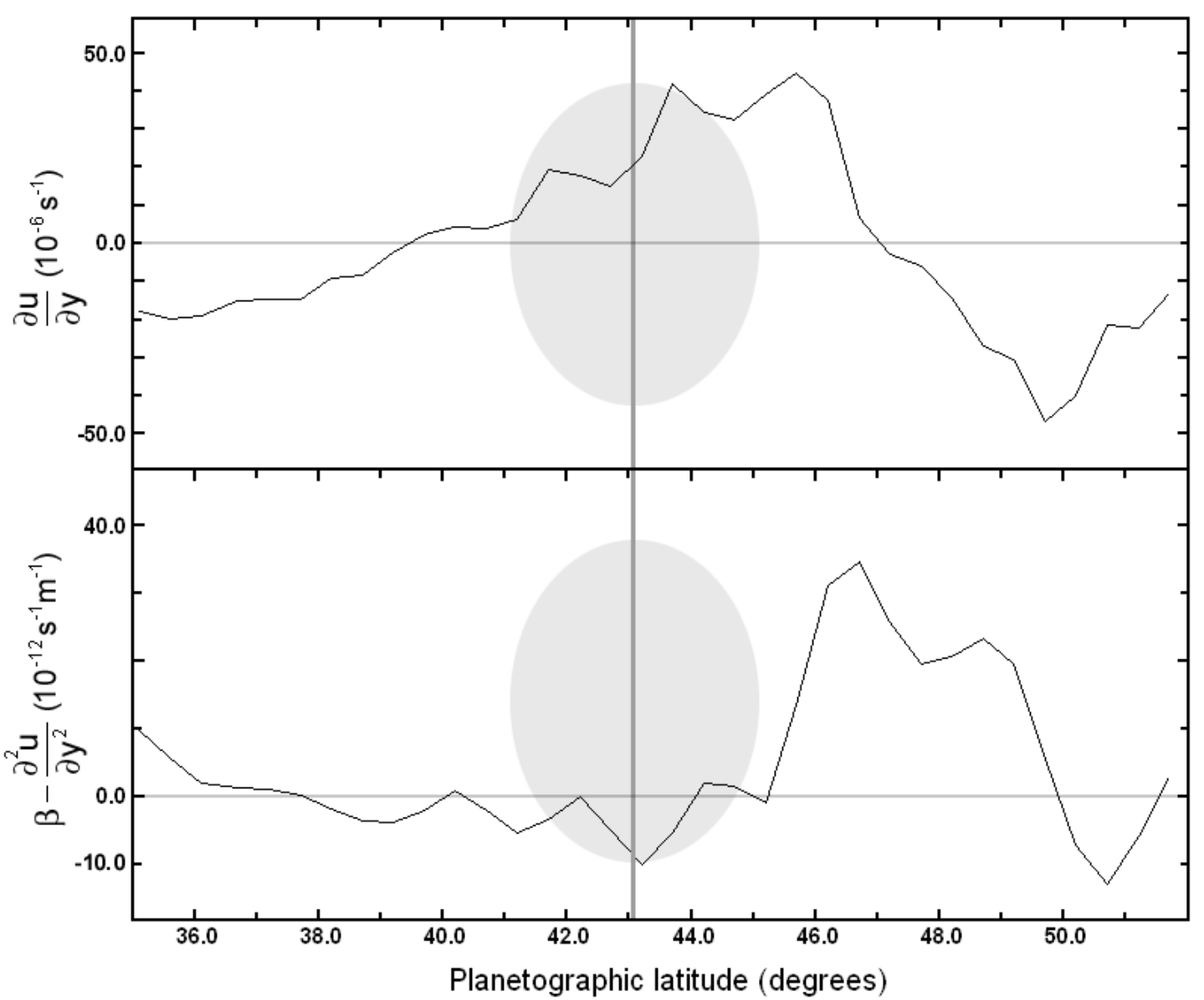

Figure 11 